Draft VERSiOn JANUARY 31, 2019

Typeset using LATEX twocolumn style in AASTeX61

\title{
EXTENDED CALCULATIONS OF ENERGY LEVELS AND TRANSITION RATES OF ND II-IV IONS FOR APPLICATION TO NEUTRON STAR MERGERS
}

Gediminas Gaigalas, ${ }^{1}$ Daiju Kato, ${ }^{2,3}$ Pavel Rynkun, ${ }^{1}$ Laima Radžıūtė,${ }^{1}$ and Masaomi Tanaka ${ }^{4}$

\footnotetext{
${ }^{1}$ Institute of Theoretical Physics and Astronomy, Vilnius University, Sauletekio Ave. 3, Vilnius, Lithuania

${ }^{2}$ National Institute for Fusion Science, 322-6 Oroshi-cho, Toki 509-5292, Japan

${ }^{3}$ Department of Advanced Energy Engineering, Kyushu University, Kasuga, Fukuoka 816-8580, Japan

${ }^{4}$ Astronomical Institute, Tohoku University, Aoba, Sendai 980-8578, Japan
}

(Received January 31, 2019; Revised; Accepted)

\begin{abstract}
Coalescence of binary neutron star give rise to electromagnetic emission, kilonova, powered by radioactive decays of $r$-process nuclei. Observations of kilonova associated with GW170817 provided unique opportunity to study the heavy element synthesis in the Universe. However, atomic data of $r$-process elements to decipher the light curves and spectral features of kilonova are not fully constructed yet. In this paper, we perform extended atomic calculations of neodymium (Nd, $Z=60$ ) to study the impact of accuracies in atomic calculations to the astrophysical opacities. By employing multiconfiguration Dirac-Hartree-Fock and relativistic configuration interaction methods, we calculate energy levels and transition data of electric dipole transitions for Nd II, Nd III, and Nd IV ions. Compared with previous calculations, our new results provide better agreement with the experimental data. The accuracy of energy levels was achieved in the present work $10 \%, 3 \%$ and $11 \%$ for Nd II, Nd III and Nd IV, respectively, comparing with the NIST database. We confirm that the overall properties of the opacity are not significantly affected by the accuracies of the atomic calculations. The impact to the Planck mean opacity is up to a factor of 1.5, which affects the timescale of kilonova at most 20\%. However, we find that the wavelength dependent features in the opacity are affected by the accuracies of the calculations. We emphasize that accurate atomic calculations, in particular for low-lying energy levels, are important to provide predictions of kilonova light curves and spectra.
\end{abstract}

Keywords: radiative transfer — opacity — stars: neutron 


\section{INTRODUCTION}

On 2017 August 18, the first observation of gravitational waves $(\mathrm{GWs})$ from neutron star (NS) merger was achieved (GW170817, Abbott et al. (2017a)). In addition to GWs, electromagnetic (EM) counterparts across the wide wavelength range were also observed (Abbott et al. 2017b). In particular, intensive observations of the optical and near-infrared (NIR) counterpart (SSS17a, also known as DLT17ck or AT2017gfo) have been performed and dense photometric and spectroscopic data were obtained (Andreoni et al. 2017; Arcavi et al. 2017; Chornock et al. 2017; Coulter et al. 2017; Cowperthwaite et al. 2017; Díaz et al. 2017; Drout et al. 2017; Evans et al. 2017; Kasliwal et al. 2017; Kilpatrick et al. 2017; Lipunov et al. 2017; McCully et al 2017; Nicholl et al. 2017; Pian et al. 2017; Shappee et al. 2017; Siebert et al. 2017; Smartt et al. 2017; Soares-Santos et aten 2017; Tanvir et al. 2017; Tominaga et al. 2018; Troja et al. 2017; Utsumi et al. 2017; Valenti et al. 2017). SSS17a shows characteristic properties that are quite different from those of supernovae. The optical light curves decline rapidly while NIR light curves evolve more slowly. The spectra show feature-less, broad-line features implying a high expansion velocity. These properties are broadly consistent with theoretically suggested kilonova or macronova emission from NS mergers (Li \& Paczyński 1998; Kulkarni 2005; Metzger et al. 2010).

Kilonova is EM emission powered by radioactive decay energy of $r$-process nuclei that are newly synthesized in the NS mergers (see Rosswog 2015; Tanaka 2016; Fernández \& Metzger 2016; Metzger 2017, for reviews). The timescale, luminosity, and color of the emission are mainly determined by the mass and velocity of the ejecta and opacities in the ejecta. Among $r$-process elements, lanthanide elements have high optical and NIR opacities (Kasen et al. 2013; Tanaka \& Hotokezaka 2013). Therefore, if the ejecta include lanthanide elements, the emission becomes red and faint. On the other hand, if the ejecta is free from lanthanide elements, the emission is blue and bright (Metzger \& Fernández 2014; Kasen et al. 2015; Tanaka et al. 2018).

In fact, SSS17a shows both blue and red components, which implies the presence of multiple components with different lanthanide contents. This fact suggests the production of a wide range of $r$-process elements (Kasen et al. 2017; Tanaka et al. 2017; Rosswog et al. 2017). This is also consistent with the expectation from numerical relativity simulations (see e.g., Shibata et al. 2017; Perego et al. 2017). The ejecta mass to explain the luminosity of SSS17a is about $0.03-0.06 M_{\odot}$. Although it is still unclear if the $r$-process yields from NS mergers are consistent with the solar ratios, NS mergers may be the dominant site for the $r$-process elements in the Universe (Rosswog et al. 2017; Hotokezaka et al. 2018).

Although the observed properties can be explained by kilonova scenario, physics included in current kilonova simulations is not yet perfect. In particular, atomic data of $r$-process elements are not complete: so far calculated data are available only for limited number of $r$ process elements (Kasen et al. 2013; Fontes et al. 2017; Wollaeger et al. 2017; Kasen et al. 2017; Tanaka et al. 2018). Even when the data are available, they are almost entirely based on theoretical calculations, and derived energy levels often deviates from experimental data by up to $\sim 30 \%$ (note that experimental data are also insufficient). It is not yet clear if these issues bring systematic impacts to the opacities as well as properties of kilonova.

In this paper, we study impacts of the accuracies in atomic calculations to the opacities by performing extensive, accurate calculations. For this purpose, we choose a lanthanide element, neodymium ( $\mathrm{Nd}, Z=60)$, which has also been studied by Kasen et al. (2013); Fontes et al. (2017); Tanaka et al. (2018). We focus on singly to triply ionized $\mathrm{Nd}$, for which accurate calculations are possible with the multiconfiguration DiracHartree-Fock method. In Sections 2 and 3, we describe methods and strategies of our atomic calculations. In Section 4, we show and evaluate results of atomic calculations. In Section 5, we show the impact of the accuracy of atomic calculations to the astrophysical opacities. Finally we give summary in Section 6 .

\section{METHODS}

\subsection{Computational procedure}

The GRASP2K package (Jönsson et al. 2013) is based on the multiconfiguration Dirac-Hartree-Fock (MCDHF) and relativistic configuration interaction (RCI) methods taking into account the transverse photon interaction (Breit interaction) and quantum electrodynamic (QED) corrections (Grant 2007; Fischer et al. 2016).

The MCDHF method used in the present work is based on the Dirac-Coulomb Hamiltonian

$$
H_{D C}=\sum_{i=1}^{N}\left(c \boldsymbol{\alpha}_{i} \cdot \boldsymbol{p}_{i}+\left(\beta_{i}-1\right) c^{2}+V_{i}^{N}\right)+\sum_{i>j}^{N} \frac{1}{r_{i j}},(1)
$$

where $V^{N}$ is the monopole part of the electron-nucleus Coulomb interaction, $\boldsymbol{\alpha}$ and $\beta$ are the $4 \times 4$ Dirac matrices, and $c$ is the speed of light in atomic units. The atomic state functions (ASF) were obtained as linear 
combinations of symmetry adapted configuration state functions (CSFs)

$$
\Psi(\gamma P J M)=\sum_{j=1}^{N C S F s} c_{j} \Phi\left(\gamma_{j} P J M\right) .
$$

Here $J$ and $M$ are the angular quantum numbers and $P$ is parity. $\gamma_{j}$ denotes other appropriate labeling of the configuration state function $j$, for example orbital occupancy and coupling scheme. Normally the label $\gamma$ of the atomic state function is the same as the label of the dominant CSF. The CSFs are built from products of one-electron Dirac orbitals. Based on a weighted energy average of several states, the so called extended optimal level (EOL) scheme (Dyall et al. 1989), both the radial parts of the Dirac orbitals and the expansion coefficients were optimized to self-consistency in the relativistic selfconsistent field procedure. Note that accurate calculations with the MCDHF method is much more difficult for neutral atoms than ions (Grant 2007), we focus on ionized $\mathrm{Nd}$.

For these calculation, we used the spin-angular approach (Gaigalas \& Rudzikas 1996; Gaigalas et al. 1997) which is based on the second quantization in coupled tensorial form, on the angular momentum theory in three spaces (orbital, spin, and quasispin) and on the reduced coefficients of fractional parentage. It allow us to study configurations with open $f$-shells without any restrictions.

In subsequent RCI calculations the Breit interaction

$$
\begin{gathered}
H_{\text {Breit }}=-\sum_{i<j}^{N}\left[\boldsymbol{\alpha}_{i} \cdot \boldsymbol{\alpha}_{j} \frac{\cos \left(\omega_{i j} r_{i j} / c\right)}{r_{i j}}\right. \\
\left.+\left(\boldsymbol{\alpha}_{i} \cdot \nabla_{i}\right)\left(\boldsymbol{\alpha}_{j} \cdot \nabla_{j}\right) \frac{\cos \left(\omega_{i j} r_{i j} / c\right)-1}{\omega_{i j}^{2} r_{i j} / c^{2}}\right]
\end{gathered}
$$

was included in the Hamiltonian. The photon frequencies $\omega_{i j}$, used for calculating the matrix elements of the transverse photon interaction, were taken as the difference of the diagonal Lagrange multipliers associated with the Dirac orbitals (McKenzie et al. 1980). In the RCI calculation the leading QED corrections, selfinteraction and vacuum polarization, were also included.

In the present calculations, the ASFs were obtained as expansions over $j j$-coupled CSFs. To provide the $L S J$ labeling system, the ASFs were transformed from a $j j$ coupled CSF basis into an $L S J$-coupled CSF basis using the method provided by Gaigalas et al. (2017).

\subsection{Computation of transition parameters}

The evaluation of radiative transition data (transition probabilities, oscillator strengths) between two states: $\gamma^{\prime} P^{\prime} J^{\prime} M^{\prime}$ and $\gamma P J M$, built on different and independently optimized orbital sets is non-trivial. The transition data can be expressed in terms of the transition moment, which is defined as

$$
\begin{array}{r}
\left\langle\Psi(\gamma P J)\|\mathbf{T}\| \Psi\left(\gamma^{\prime} P^{\prime} J^{\prime}\right)\right\rangle= \\
\sum_{j, k} c_{j} c_{k}^{\prime}\left\langle\Phi\left(\gamma_{j} P J\right)\|\mathbf{T}\| \Phi\left(\gamma_{k}^{\prime} P^{\prime} J^{\prime}\right)\right\rangle,
\end{array}
$$

where $\mathbf{T}$ is the transition operator. For electric dipole and quadrupole (E1 and E2) transitions there are two forms of the transition operator: the length (Babushkin) and velocity (Coulomb) forms, which for the exact solutions of the Dirac-equation give the same value of the transition moment (Grant 1974). The quantity $d T$, characterizing the accuracy of the computed transition rates, is defined as

$$
d T=\frac{\left|A_{1}-A_{\mathrm{v}}\right|}{\max \left(A_{1}, A_{\mathrm{v}}\right)}
$$

where $A_{1}$ and $A_{\mathrm{v}}$ are transition rates in length and velocity forms.

The calculation of the transition moment breaks down to the task of summing up reduced matrix elements between different CSFs. The reduced matrix elements can be evaluated using standard techniques assuming that both left and right hand CSFs are formed from the same orthonormal set of spin-orbitals. This constraint is severe, since a high-quality and compact wave function requires orbitals optimized for a specific electronic state, see for example (Fritzsche \& Grant 1994). To get around the problems of having a single orthonormal set of spin-orbitals, the wave function representations of the two states: $\gamma^{\prime} P^{\prime} J^{\prime} M^{\prime}$ and $\gamma P J M$, were transformed in such way that the orbital sets became biorthonormal (Olsen et al. 1995). Standard methods were then used to evaluate the matrix elements of the transformed CSFs.

\section{SCHEMES OF THE CALCULATIONS}

\subsection{Active space construction}

Summary of the MCDHF and RCI calculations for each ion is given in Table 1. The description, which explains in what way these calculations were done is given below. As a starting point DHF calculations were performed in the EOL scheme for the states of the ground configuration. The wave functions from these calculations were taken as the initial ones to calculate even and odd states of multireference (MR) configurations. The set of orbitals belonging to these MR configurations are referred to as 0 layer $(0 \mathrm{~L})$. 
Table 1. Summary of active space construction

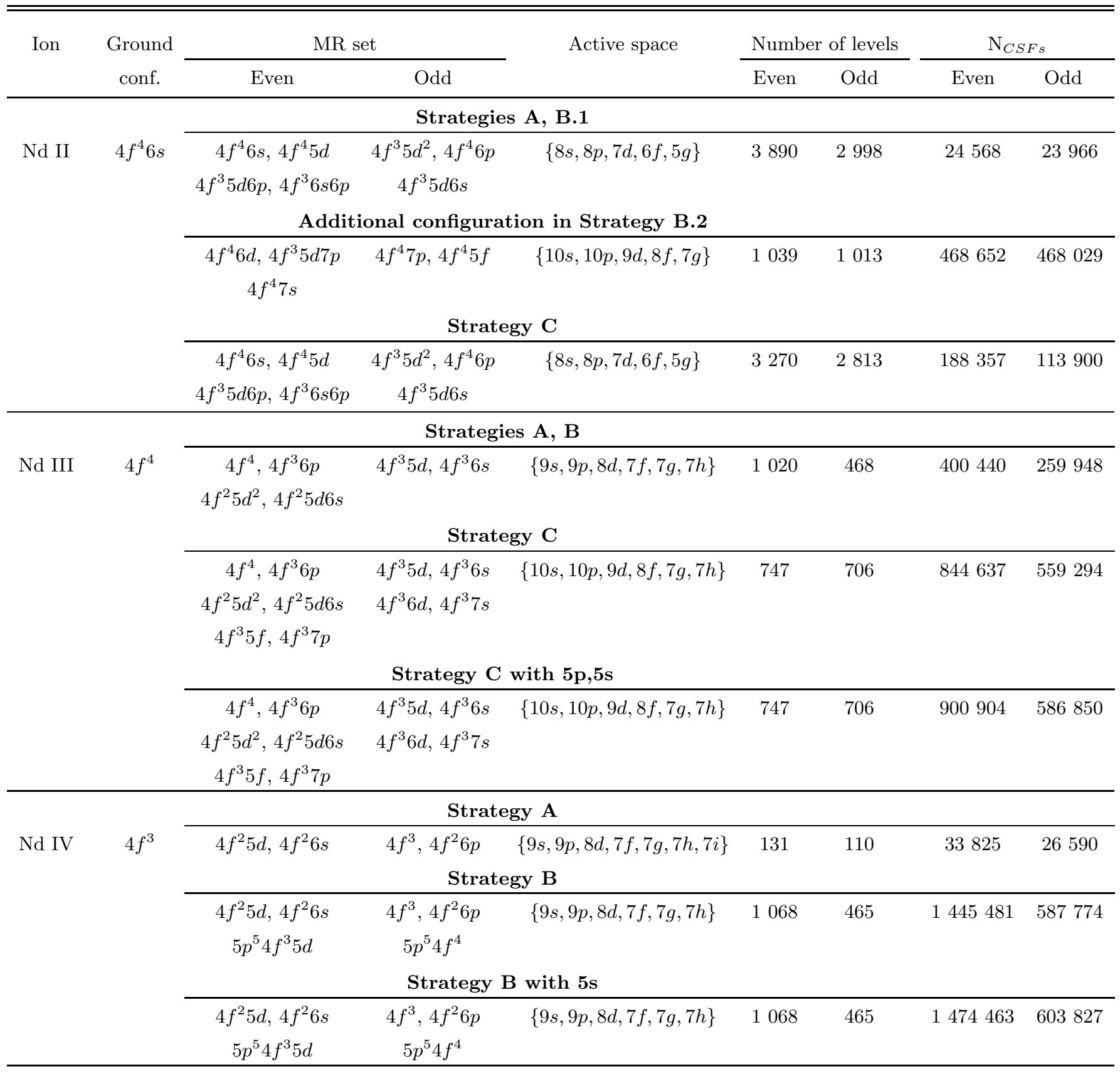

Unless stated otherwise, the inactive core of each ion used in present calculations is $[\mathrm{Xe}]$. The CSF expansions for states of each parity were obtained by allowing single $(\mathrm{S})$ and double (SD) substitutions from the MR configurations up to active orbital sets (see Table 1). The configuration space was increased step by step with increasing the number of layers (L). The orbitals of previous layers were held fixed and only the orbitals of the newest layer were allowed to vary. For example, the scheme used to increase the active spaces of the CSFs for Nd III ion (in Strategy A) is presented below:

$\mathrm{AS}_{0 L}=\{6 \mathrm{~s}, 6 \mathrm{p}, 5 \mathrm{~d}\}$,

$\mathrm{AS}_{1 L}=\mathrm{AS}_{0 L}+\{7 \mathrm{~s}, 7 \mathrm{p}, 6 \mathrm{~d}, 5 \mathrm{f}, 5 \mathrm{~g}\}$,

$\mathrm{AS}_{2 L}=\mathrm{AS}_{1 L}+\{8 \mathrm{~s}, 8 \mathrm{p}, 7 \mathrm{~d}, 6 \mathrm{f}, 6 \mathrm{~g}, 6 \mathrm{~h}\}$,

$\mathrm{AS}_{3 L}=\mathrm{AS}_{2 L}+\{9 \mathrm{~s}, 9 \mathrm{p}, 8 \mathrm{~d}, 7 \mathrm{f}, 7 \mathrm{~g}, 7 \mathrm{~h}\}$.

The MCDHF calculations were followed by RCI calculations, including the Breit interaction and leading QED effects. The number of CSFs in the final even and odd 
state expansions distributed over the different $J$ symmetries is presented in Table 1.

\subsection{Strategies for $N d I I$ ion}

Four strategies were tested for Nd II ion. All of them were computed in the active space described in the Table 1. For the Strategy A a starting point DHF calculations were performed in the EOL scheme for the states of the ground configuration $4 f^{4} 6 s$. The wave functions from these calculations were taken as the initial ones to calculate even and odd states of MR configurations. The set of orbitals belonging to these MR configurations are referred to as 0 layer $(0 \mathrm{~L})$. The active space were generated as is presented in the Table 1.

For Strategy B.1 the starting point was computation of the wave functions for the core $4 f^{4} 6 s$. Wave functions were computed in the neutral system of $\mathrm{Nd}$ I - ground state $4 f^{4} 6 s^{2}$. Then $\mathrm{AS}_{0 L}$ was computed: the core shells were frozen and only $5 d$ and $6 p$ shells of the configurations of MR listed in Table 1 were computed. Even and odd states were computed together. Later, wave functions were optimized separately for states of different parities in the $\mathrm{AS}_{1 L} \cdot \mathrm{AS}_{1 L}$ and the next active space were generated by SD substitutions from shells $4 f, 5 d, 6 p, 6 s$.

In the Strategy B.2 the configurations of the Rydberg states listed in Table 1 were added to the multireference list; therefore, the first active set included subshells bigger by one principal quantum number. Then the first active space of the Strategy B.2 was $\mathrm{AS}_{1 L}=$ $\mathrm{AS}_{0 L}+\{8 \mathrm{~s}, 8 \mathrm{p}, 7 \mathrm{~d}, 6 \mathrm{f}, 5 \mathrm{~g}\}$.

In Strategy $\mathbf{C}$ computation were performed for each configuration separately. For configurations $4 f^{4} 6 s$, $4 f^{4} 6 p$ and $4 f^{4} 5 d \mathrm{SD}$ substitutions were allowed from $4 f^{4} n l$ (where $l=s, p, d$ ) shells in to the $A S_{0 L, 1 L}$ and $\mathrm{S}$ to the $A S_{2 L}$. For configurations $4 f^{3} 5 d 6 s, 4 f^{3} 5 d 6 p$, $4 f^{3} 6 s 6 p$, and $4 f^{3} 5 d^{2}$ only $\mathrm{S}$ substitutions were allowed. Radial wavefunctions up to $4 f$ orbital was taken from ground configuration for these configurations. The Breit interaction and leading QED effects are included in RCI computations.

\subsection{Strategies for $N d$ III ion}

After $\mathrm{AS}_{0 L}$ the even and odd states were calculated separately in Strategy A. For the Nd III ion calculations the Strategy B was also applied. Strategy B differs from Strategy A in the fact that virtual orbitals for odd parity were taken from even parity states instead of varying them in layer 1 , and higher layers.

In Strategy $\mathbf{C}$ as compared to Strategy $\mathbf{A}$ additional configurations: $4 f^{3} 6 d, 4 f^{3} 7 s$ (odd parity) and $4 f^{3} 5 f, 4 f^{3} 7 p$ (even parity) were added to the MR set.
In Strategy C with $5 p, 5 s$ just RCI calculations were performed. The wavefunctions were taken from Strategy $\mathbf{C}$ and configurations with $\mathbf{S}$ substitutions from $5 p$ and $5 s$ shells to $\{6 s, 6 p, 5 d, 4 f\}$ shells were added additionally in the active space.

\subsection{Strategies for $N d I V$ ion}

In Strategy B as compared to Strategy A additional configurations: $4 p^{5} 4 f^{4}$ (odd parity) and $4 p^{5} 4 f^{3} 5 d$ (even parity) were added to the MR set. The AS for even and odd parities were constructed in such way: SD substitutions were allowed from the $4 f, 5 d, 6 s, 6 p$ shells up to active orbital sets and S substitution from $5 p$ shell to $\{6 s, 6 p, 5 d, 4 f\}$ shells. In Strategy B with $\mathbf{5 s}$ just RCI calculations were performed. The wave functions were taken from Strategy B and configurations with S substitutions from $5 s$ shells to $\{6 s, 6 p, 5 d, 4 f\}$ shells were added additionally in the active space.

\section{RESULTS}

\subsection{NdII}

A part of all computed excitation energies for $\mathrm{Nd}$ II are listed in Table 2. These data were compared with NIST database by evaluating relative difference $\Delta E / E=\left(E_{N I S T}-E\right) / E_{N I S T}$. Energy levels computed with Breit interaction and QED effects are presented in columns marked by *. Levels with changed notations are given in Table 3 .

Note that the energy levels of Nd II are also provided by Wyart (2010). They interpreted 596 levels of odd configurations $\left(4 f^{3} 5 d 6 s, 4 f^{3} 5 d^{2}, 4 f^{3} 6 s^{2}, 4 f^{4} 6 p\right.$ and $4 f^{5}$ ) in semi-empirical way following the RacahSlater parametric method, by using the Cowan computer codes. In their method, radial parameters obtained in a least-squares fit were compared with HartreeFock (HFR) ab initio integrals. In such a way, obtained energy levels naturally have very small disagreement with NIST values, therefore are not presented in this paper.

Energy levels for each configurations are compared with NIST in the figure 1. Among different strategies, Strategy C $A S_{2 L}$ gives the best agreement with the NIST database. Averaged difference between our computed data and NIST presented values is $10 \%$. This is significant improvement as compared with Strategy A $A S_{2 L}$ (blue in Figure 1), which was used to compute the opacity of the neutron star mergers in Tanaka et al. (2018). The averaged difference with the NIST is 22 $\%$ in Strategy A $A S_{2 L}$. For the comparison with the NIST, expression $\overline{\Delta E / E}=\frac{\sum\left|\Delta E_{i} / E_{i}\right|}{N}$ was used, where $N$ is the number of compared levels. 


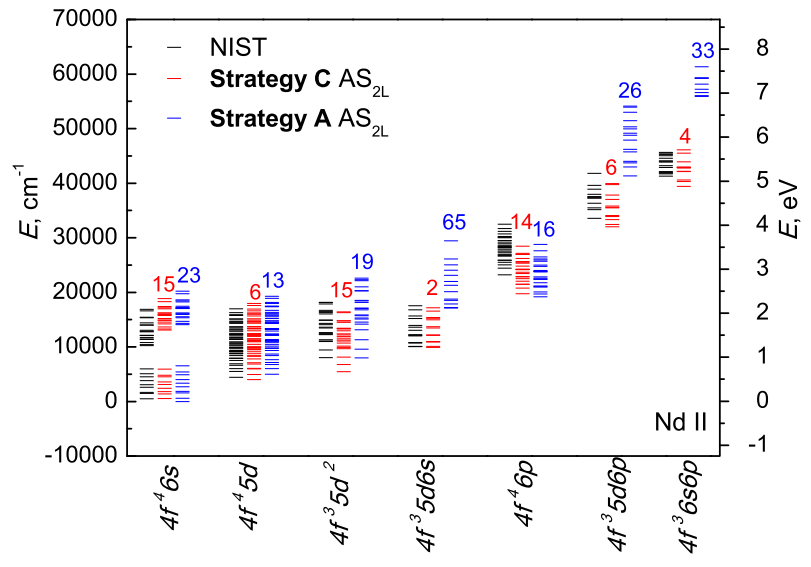

Figure 1. Energy levels for configurations of Nd II are compared with data of NIST. Black color is representing NIST data, next column of levels in red is our computed energy levels in Strategy C $A S_{2 L}$, blue color data are based on Startegy A $A S_{2 L}$ (used in Tanaka et al. 2018). Number on top of red and blue column is averaged disagreement in \% for levels of each configuration comparing with NIST database.
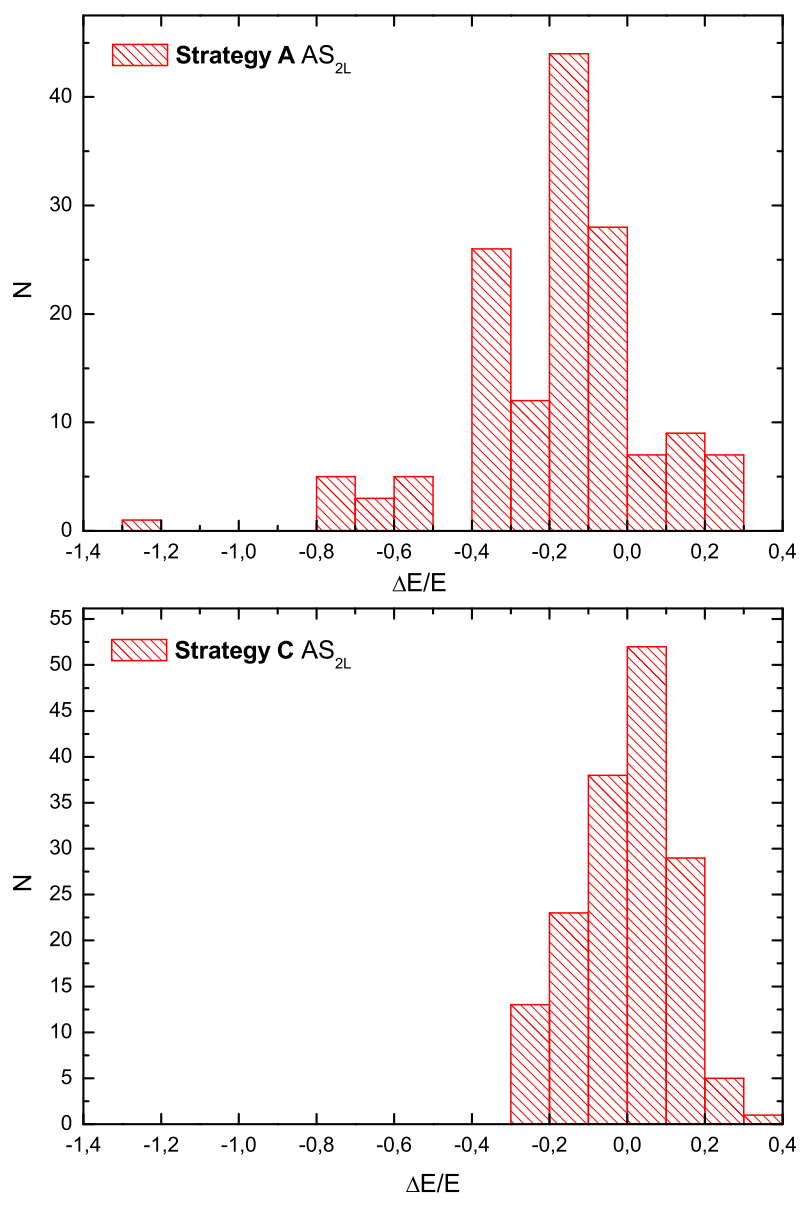

Figure 2. Distribution of the energy levels (N) according the disagreement with NIST database for Nd II using Strategy A and Strategy $\mathbf{C}$ in $A S_{2 L}$.
The figure 2 shows distribution of the energy levels number over relative difference comparing with the NIST for Strategy $\mathbf{A}$ in active space $A S_{2} L$. For the strategies A, B.1 and B.2 in all active spaces the view of the distribution is very similar. In case of Strategy $\mathrm{C}$ in $A S_{2}$ (see figure 2) normal distribution with smaller $\Delta E / E$ range is observed.

Energy data computed in Strategy $\mathbf{C}$ at layer 2 are given in machine readable Table 9. This includes number, label, $J$ and $P$ values, and energy value. Transitions data obtained from Strategy $\mathbf{C}$ at layer 2 are given in machine readable Table 10. This includes identification of upper and lower levels in $L S J$ coupling, transition energy, wavelength, line strength, weighted oscillator strength, and transitions probabilities in length form.

\section{2. $N d I I I$}

Results of the energy levels for Nd III obtained applying Strategies: A, B C, and C with 5p, 5s are compared with the NIST database and presented in Table 4. Among different strategies, Strategy C with 5p, $\mathbf{5 s}$ gives the best agreement with the NIST database although the number of availabe levels is smaller than in the case of Nd II. All the energy levels and transition data obtained from this strategy are given the machine readable Tables 11 and 12 . Figure 3 shows the comparison of the energy levels with the NIST database. The averaged difference between our calculations with Strategy $\mathbf{C}$ with $\mathbf{5 p}, \mathbf{5 s} A S_{3 L}$ and the NIST data is 3 $\%$. For comparison, the difference for the case of Strategy B $A S_{2 L}$, which as used by Tanaka et al. (2018), is $5 \%$ (blue in Figure 3).

Results of the energy levels obtained from Strategy $\mathbf{C}$ with $5 p, 5 s$ are also compared with those by Dzuba et al. (2003) in Table 5. They evaluated energy levels and lifetimes of configurations $4 f^{4}, 4 f^{3} 5 d$ using relativistic Hartree-Fock and configuration-interaction (RCI) codes as well as a set of computer codes written by Cowan (1981). Note that Zhang, Z. G. et al. (2002) also presented low-lying odd energy levels (below $33000 \mathrm{~cm}^{-1}$ ) belonging to the configurations: $4 f^{3} 5 d$ and $4 f^{3} 6 s$. To compute these energy levels, the HFR, described and coded by Cowan (1981) but modified with the inclusion of core-polarization effects was used. It should be mentioned, however, that core-core correlation was not included in energy levels computations.

Disagreement between our data obtained applying Strategy C and Strategy C with 5p, 5s as compared to recommended data by NIST is slightly larger than disagreement between data computed by Dzuba et al. (2003) (Cowan) as compared to recommended data by NIST. In this paper we present the lowest 1453 levels 
Table 2. Computations of energy values (in $\mathrm{cm}^{-1}$ ) of Nd II by increase of the active space performed applying Strategy A, Strategy B.1, Strategy B.2, and Strategy C and their comparison with NIST data base (in \%). With the '*, energy levels were computed with Breit interaction and QED effects are presented.

\begin{tabular}{|c|c|c|c|c|c|c|c|c|c|c|c|c|c|}
\hline \multirow{3}{*}{$\frac{\text { Config. }}{4 f^{4}\left({ }^{5} I\right) 6 s}$} & \multirow{3}{*}{$\frac{\text { Term }}{{ }^{6} I}$} & \multirow{3}{*}{$\frac{J}{7 / 2}$} & \multirow{3}{*}{$\begin{array}{c}\text { NIST } \\
0.000\end{array}$} & \multicolumn{2}{|c|}{ Strategy A } & \multicolumn{3}{|c|}{ Strategy B.1 } & \multicolumn{3}{|c|}{ Strategy B.2 } & \multicolumn{2}{|c|}{ Strategy C } \\
\hline & & & & $\mathrm{AS}_{1 L} /$ & $\overline{\mathrm{AS}_{2 L}}$ & \multicolumn{3}{|c|}{$\mathrm{AS}_{1 L} / \mathrm{AS}_{2 L} / \mathrm{AS}_{2 L} * / \mathrm{AS}_{3 L}$} & \multicolumn{3}{|c|}{$\mathrm{AS}_{1-2 L} / \mathrm{AS}_{2-3 L} / \mathrm{AS}_{3-4 L}$} & \multicolumn{2}{|c|}{$\mathrm{AS}_{1 L} * / \mathrm{AS}_{2 L} *$} \\
\hline & & & & & & & & & & & & & \\
\hline & ${ }^{6} I$ & $9 / 2$ & 513.330 & $-14 /$ & -9 & $-16 /$ & $-10 /$ & $-2 /-10$ & $-9 /$ & $-9 /$ & -9 & $-6 /$ & -7 \\
\hline & ${ }^{6} I$ & $11 / 2$ & 1470.105 & $-5 /$ & -3 & $-6 /$ & $-3 /$ & $8 / \quad-3$ & $-0 /$ & $0 /$ & -3 & $5 /$ & 4 \\
\hline $4 f^{4}\left({ }^{5} I\right) 6 s$ & ${ }^{4} I$ & $9 / 2$ & 1650.205 & $-13 /$ & -8 & $-15 /$ & $-9 /$ & $-3 / \quad-8$ & $-8 /$ & $-7 /$ & -8 & $-8 /$ & -8 \\
\hline $4 f^{4}\left({ }^{5} I\right) 6 s$ & ${ }^{6} I$ & $13 / 2$ & 2585.460 & $-5 /$ & -3 & $-4 /$ & $-3 /$ & $9 / \quad-3$ & $1 /$ & $1 /$ & -3 & $6 /$ & 6 \\
\hline $4 f^{4}\left({ }^{5} I\right) 6 s$ & ${ }^{4} I$ & $11 / 2$ & 3066.755 & $-9 /$ & -6 & $-10 /$ & $-6 /$ & $2 / \quad-6$ & $-4 /$ & $-4 /$ & -6 & $-1 /$ & -2 \\
\hline $4 f^{4}\left({ }^{5} I\right) 6 s$ & ${ }^{6} I$ & $15 / 2$ & 3801.930 & $-5 /$ & -4 & $-5 /$ & $-4 /$ & $8 /-4$ & $0 /$ & $0 /$ & -4 & $6 /$ & 6 \\
\hline $4 f^{4}\left({ }^{5} I\right) 5 d$ & ${ }^{6} L$ & $11 / 2$ & 4437.560 & $-13 /$ & -7 & $-38 /$ & $-3 /$ & $2 / \quad-3$ & $-2 /$ & $-3 /$ & 1 & $4 /$ & 9 \\
\hline $4 f^{4}\left({ }^{5} I\right) 6 s$ & ${ }^{4} I$ & $13 / 2$ & 4512.495 & $-9 /$ & -6 & $-9 /$ & $-6 /$ & $0 / \quad-6$ & $-4 /$ & $-3 /$ & -6 & $0 /$ & 0 \\
\hline $4 f^{4}\left({ }^{5} I\right) 6 s$ & ${ }^{6} I$ & $17 / 2$ & 5085.640 & $-6 /$ & -5 & $-6 /$ & $-5 /$ & $7 / \quad-5$ & $-1 /$ & $-1 /$ & -5 & $6 /$ & 6 \\
\hline $4 f^{4}\left({ }^{5} I\right) 5 d$ & ${ }^{6} L$ & $13 / 2$ & 5487.655 & $-10 /$ & -5 & $-30 /$ & $-2 /$ & $1 /-2$ & $-1 /$ & $-1 /$ & 1 & $6 /$ & 10 \\
\hline $4 f^{4}\left({ }^{5} I\right) 6 s$ & ${ }^{4} I$ & $15 / 2$ & 5985.580 & $-9 /$ & -7 & $-9 /$ & $-7 /$ & $4 / \quad-7$ & $-4 /$ & $-4 /$ & -7 & $1 /$ & 1 \\
\hline $4 f^{4}\left({ }^{5} I\right) 5 d$ & ${ }^{6} K$ & $9 / 2$ & 6005.270 & $-12 /$ & -7 & $-36 /$ & $-4 /$ & $-3 / \quad-4$ & $-5 /$ & $-5 /$ & -2 & $-6 /$ & -1 \\
\hline $4 f^{4}\left({ }^{5} I\right) 5 d$ & ${ }^{6} L$ & $15 / 2$ & 6637.430 & $-8 /$ & -4 & $-24 /$ & $-1 /$ & $3 /-1$ & $0 /$ & $0 /$ & 0 & $7 /$ & 10 \\
\hline $4 f^{4}\left({ }^{5} I\right) 5 d$ & ${ }^{6} K$ & $11 / 2$ & 6931.800 & $-10 /$ & -6 & $-31 /$ & $-3 /$ & $-1 / \quad-3$ & $-3 /$ & $-3 /$ & -2 & $-3 /$ & 1 \\
\hline $4 f^{4}\left({ }^{5} I\right) 5 d$ & ${ }^{6} I$ & $7 / 2$ & 7524.735 & $-24 /$ & -19 & $-50 /$ & $-17 /$ & $-16 /-17$ & $-19 /$ & $-19 /$ & -15 & $-16 /$ & -1 \\
\hline $4 f^{4}\left({ }^{5} I\right) 5 d$ & ${ }^{6} L$ & $17 / 2$ & 7868.910 & $-7 /$ & -3 & $-20 /$ & $-1 /$ & $4 / \quad-1$ & $1 /$ & $1 /$ & 0 & $7 /$ & 10 \\
\hline $4 f^{4}\left({ }^{5} I\right) 5 d$ & ${ }^{6} K$ & $13 / 2$ & 7950.075 & $-9 /$ & -5 & $-27 /$ & $-3 /$ & $1 /-3$ & $-3 /$ & $-3 /$ & -2 & $-2 /$ & 2 \\
\hline $4 f^{3}\left({ }^{4} I\right) 5 d^{2}\left({ }^{3} F\right)$ & ${ }^{6} M^{o}$ & $13 / 2$ & 8009.810 & $0 /$ & -12 & $-7 /$ & $-11 /$ & $-6 /-14$ & $-7 /$ & $-10 /$ & -14 & $32 /$ & 32 \\
\hline $4 f^{4}\left({ }^{5} I\right) 5 d$ & ${ }^{6} I$ & $9 / 2$ & 8420.320 & $-21 /$ & -16 & $-43 /$ & $-14 /$ & $-12 /-14$ & $-15 /$ & $-16 /$ & -13 & $-13 /$ & -9 \\
\hline \multirow[t]{2}{*}{$4 f^{4}\left({ }^{5} I\right) 5 d$} & ${ }^{6} G$ & $3 / 2$ & 8716.445 & $-20 /$ & -15 & $-42 /$ & $-13 /$ & $-12 /-13$ & $-16 /$ & $-16 /$ & -13 & $-14 /$ & -11 \\
\hline & ${ }^{6} G$ & $5 / 2$ & 8796.365 & $-23 /$ & -17 & $-46 /$ & $-15 /$ & $-14 /-15$ & $-19 /$ & $-19 /$ & -15 & $-17 /$ & -14 \\
\hline $4 f^{4}\left({ }^{5} I\right) 5 d$ & ${ }^{6} K$ & $15 / 2$ & 9042.760 & $-8 /$ & -5 & $-24 /$ & $-3 /$ & $2 / \quad-3$ & $-2 /$ & $-2 /$ & -2 & $0 /$ & 3 \\
\hline $4 f^{4}\left({ }^{5} I\right) 5 d$ & ${ }^{6} L$ & $19 / 2$ & 9166.210 & $-6 /$ & -3 & $-17 /$ & $-1 /$ & $5 /-1$ & $1 /$ & $1 /$ & -1 & $7 /$ & 10 \\
\hline $4 f^{4}\left({ }^{5} I\right) 5 d$ & ${ }^{6} G$ & $7 / 2$ & 9198.395 & $-24 /$ & -18 & $-46 /$ & $-16 /$ & $-15 /-16$ & $-19 /$ & $-19 /$ & -15 & $-17 /$ & -14 \\
\hline $4 f^{4}\left({ }^{5} I\right) 5 d$ & ${ }^{6} I$ & $11 / 2$ & 9357.910 & $-18 /$ & -14 & $-39 /$ & $-12 /$ & $-10 /-12$ & $-13 /$ & $-13 /$ & -11 & $-10 /$ & -7 \\
\hline $4 f^{3}\left({ }^{4} I\right) 5 d^{2}\left({ }^{3} F\right)$ & ${ }^{6} M^{o}$ & $15 / 2$ & 9448.185 & $-1 /$ & -12 & $-9 /$ & $-12 /$ & $-6 /-14$ & $-8 /$ & $-11 /$ & -14 & $29 /$ & 29 \\
\hline $4 f^{4}\left({ }^{5} I\right) 5 d$ & ${ }^{6} H$ & $5 / 2$ & 9674.835 & $-28 /$ & -22 & $-55 /$ & $-20 /$ & $-20 /-30$ & $-24 /$ & $-25 /$ & -19 & $-23 /$ & -19 \\
\hline $4 f^{3}\left({ }^{4} I\right) 5 d\left({ }^{5} L\right) 6 s$ & ${ }^{6} L^{o}$ & $11 / 2$ & 10054.195 & $-71 /$ & -50 & $-73 /$ & $-77 /$ & $73 /-79$ & $-73 /$ & $-75 /$ & -79 & $1 /$ & 1 \\
\hline $4 f^{3}\left({ }^{4} I\right) 5 d\left({ }^{5} K\right) 6 s$ & ${ }^{6} K^{o}$ & $9 / 2$ & 10091.360 & $-70 /$ & -51 & $-71 /$ & $-75 /$ & $-101 /-77$ & $-71 /$ & $-73 /$ & -77 & $-1 /$ & 0 \\
\hline $4 f^{4}\left({ }^{5} I\right) 5 d$ & ${ }^{6} K$ & $17 / 2$ & 10194.805 & $-8 /$ & -5 & $-21 /$ & $-3 /$ & $2 / \quad-3$ & $-2 /$ & $-2 /$ & -3 & $1 /$ & 3 \\
\hline $4 f^{4}\left({ }^{5} F\right) 6 s$ & ${ }^{6} F$ & $1 / 2$ & 10256.040 & $-37 /$ & -37 & $-38 /$ & $-37 /$ & $-36 /-35$ & $-37 /$ & $-35 /$ & -35 & $-28 /$ & -28 \\
\hline $4 f^{4}\left({ }^{5} I\right) 5 d$ & ${ }^{6} I$ & $13 / 2$ & 10337.100 & $-17 /$ & -13 & $-35 /$ & $-11 /$ & $-8 /-11$ & $-12 /$ & $-12 /$ & -11 & $-8 /$ & -6 \\
\hline $4 f^{4}\left({ }^{5} F\right) 6 s$ & ${ }^{6} F$ & $3 / 2$ & 10439.225 & $-37 /$ & -36 & $-37 /$ & $-48 /$ & $-36 /-35$ & $-36 /$ & $-34 /$ & -35 & $-28 /$ & -27 \\
\hline $4 f^{4}\left({ }^{5} I\right) 5 d$ & ${ }^{6} L$ & $21 / 2$ & 10516.790 & $-6 /$ & -3 & $-15 /$ & $-1 /$ & $5 / \quad-1$ & $0 /$ & $0 /$ & -2 & $7 /$ & 9 \\
\hline $4 f^{4}\left({ }^{5} I\right) 5 d$ & ${ }^{6} \mathrm{H}$ & $7 / 2$ & 10666.780 & $-25 /$ & -20 & $-36 /$ & $-19 /$ & $-17 /-18$ & $-11 /$ & $-11 /$ & -8 & $-19 /$ & -16 \\
\hline $4 f^{3}\left({ }^{4} I\right) 5 d(5 K) 6 s$ & ${ }^{6} K^{o}$ & $11 / 2$ & 10720.295 & $-67 /$ & -82 & $-82 /$ & $-82 /$ & $-69 /-75$ & $-69 /$ & $-80 /$ & -75 & $-3 /$ & -2 \\
\hline $4 f^{4}\left({ }^{5} F\right) 6 s$ & ${ }^{6} F$ & $5 / 2$ & 10786.775 & $-36 /$ & -36 & $-37 /$ & $-50 /$ & $-35 /-34$ & $-35 /$ & $-47 /$ & -34 & $-27 /$ & -27 \\
\hline
\end{tabular}


Table 3. NIST recommended energy levels notations changed by the authors for the Nd II.

\begin{tabular}{|c|c|}
\hline NIST label & Our label \\
\hline $4 f^{3}\left({ }^{4} I *\right) 5 d\left({ }^{5} K *\right) 6 s{ }^{6} L *$ & $4 f^{3}\left({ }^{4} I *\right) 5 d\left({ }^{5} L *\right) 6 s{ }^{6} L *$ \\
\hline $4 f^{2}\left({ }^{4} I *\right) 5 d\left({ }^{5} K *\right) 6 s{ }^{6} I *$ & $4 f^{3}\left({ }^{4} I *\right) 5 d\left({ }^{5} I *\right) 6 s{ }^{6} I *$ \\
\hline
\end{tabular}

of energy spectra and transitions between these states whereas Dzuba et al. (2003) presented only small part of the spectra (88 levels). This paper aims at presenting a more complete set of atomic data for astrophysics. This is clearly reflected in the figure 3 where energy levels for each configurations at different strategies are presented and compared with only a few levels of configuration $4 f^{4}$ and $4 f^{3} 5 d$ available in the NIST. 
Table 4. Comparison of energy levels with NIST database (in \%) of Nd III by increase of the active space performed applying Strategies: A, B, C, and $\mathbf{C}$ with $\mathbf{5 p}, \mathbf{5 s}$. States marked by subscript ${ }^{*}$ in term column are without term identification in the NIST database.

\begin{tabular}{|c|c|c|c|c|c|c|c|c|c|c|c|c|c|c|c|}
\hline \multirow[b]{2}{*}{ Config. } & \multirow[b]{2}{*}{ Term } & \multirow[b]{2}{*}{$J$} & \multirow[b]{2}{*}{ NIST } & \multicolumn{3}{|c|}{ Strategy A } & \multicolumn{3}{|c|}{ Strategy B } & \multicolumn{3}{|c|}{ Strategy $\mathbf{C}$} & \multicolumn{3}{|c|}{ Strategy C (5p,5s) } \\
\hline & & & & $\mathrm{AS}_{1 L} /$ & $\mathrm{AS}_{2 L} /$ & $\mathrm{AS}_{3 L}$ & $\mathrm{AS}_{1 L} /$ & $\mathrm{AS}_{2 L} /$ & $\mathrm{AS}_{3 L}$ & $\mathrm{AS}_{1 L} /$ & $\mathrm{AS}_{2 L} /$ & $\mathrm{AS}_{3 L}$ & $\mathrm{AS}_{1 L} /$ & $\mathrm{AS}_{2 L} /$ & $\mathrm{AS}_{3 L}$ \\
\hline \multirow[t]{5}{*}{$4 f^{4}$} & ${ }^{5} I$ & 4 & 0.0 & & & & & & & & & & & & \\
\hline & & 5 & 1137.8 & $8.8 /$ & $8.5 /$ & 8.4 & $8.7 /$ & $8.5 /$ & 8.4 & $8.8 /$ & $8.5 /$ & 8.4 & $6.1 /$ & $5.9 /$ & 5.7 \\
\hline & & 6 & 2387.6 & $7.9 /$ & $7.7 /$ & 7.7 & $7.9 /$ & $7.7 /$ & 7.7 & $7.9 /$ & $7.8 /$ & 7.7 & $5.4 /$ & $5.3 /$ & 5.2 \\
\hline & & 7 & 3714.9 & $7.0 /$ & $7.0 /$ & 7.0 & $7.0 /$ & $7.0 /$ & 7.0 & $7.1 /$ & $7.1 /$ & 7.0 & $4.7 /$ & $4.7 /$ & 4.6 \\
\hline & & 8 & 5093.3 & $6.2 /$ & $6.2 /$ & 6.3 & $6.2 /$ & $6.2 /$ & 6.3 & $6.3 /$ & $6.4 /$ & 6.3 & $4.0 /$ & $4.1 /$ & 4.1 \\
\hline \multirow[t]{3}{*}{$4 f^{3}\left({ }^{4} I\right) 5 d$} & ${ }^{5} K^{o}$ & 5 & 15262.2 & $7.2 /$ & $11.6 /$ & 7.4 & $6.8 /$ & $6.4 /$ & 6.8 & $7.5 /$ & $7.0 /$ & 6.8 & $1.4 /$ & $1.1 /$ & 0.9 \\
\hline & & 6 & 16938.1 & $7.0 /$ & $11.0 /$ & 7.1 & $6.7 /$ & $6.3 /$ & 6.6 & $7.4 /$ & $6.8 /$ & 6.6 & $1.9 /$ & $1.5 /$ & 1.3 \\
\hline & & 7 & 18656.3 & $6.6 /$ & $10.2 /$ & 6.7 & $6.4 /$ & $6.0 /$ & 6.3 & $7.0 /$ & $6.4 /$ & 6.2 & $2.1 /$ & $1.7 /$ & 1.5 \\
\hline $4 f^{3}\left({ }^{4} I\right) 5 d$ & ${ }^{5} I^{o}$ & 4 & 18883.7 & $-2.7 /$ & $2.1 /$ & -1.2 & $-3.5 /$ & $-2.8 /$ & -1.8 & $-2.3 /$ & $-1.7 /$ & -1.7 & $0.5 /$ & $0.9 /$ & 0.9 \\
\hline \multirow[t]{2}{*}{$4 f^{3}\left({ }^{4} I\right) 5 d$} & ${ }^{5} H^{o}$ & 3 & 19211.0 & $-5.3 /$ & $-0.5 /$ & -3.8 & $-6.2 /$ & $-5.1 /$ & -4.3 & $-5.0 /$ & $-4.2 /$ & -4.2 & $-3.7 /$ & $-2.8 /$ & -2.8 \\
\hline & & 4 & 20144.3 & $-3.2 /$ & $1.3 /$ & -1.8 & $-3.9 /$ & $-3.1 /$ & -2.4 & $-2.9 /$ & $-2.3 /$ & -2.3 & $-2.3 /$ & $-1.6 /$ & -1.6 \\
\hline $4 f^{3}\left({ }^{4} I\right) 5 d$ & ${ }^{5} I^{o}$ & 5 & 20388.9 & $-1.9 /$ & $2.5 /$ & -0.6 & $-2.6 /$ & $-2.1 /$ & -1.1 & $-1.5 /$ & $-1.1 /$ & -1.1 & $0.5 /$ & $0.9 /$ & 0.9 \\
\hline $4 f^{3}\left({ }^{4} I\right) 5 d$ & ${ }^{5} K^{o}$ & 8 & 20410.9 & $6.1 /$ & $9.4 /$ & 6.2 & $5.9 /$ & $5.5 /$ & 5.8 & $6.4 /$ & $5.9 /$ & 5.7 & $2.0 /$ & $1.7 /$ & 1.5 \\
\hline $4 f^{3}\left({ }^{4} I\right) 5 d$ & ${ }^{5} H^{o}$ & 5 & 21886.8 & $-2.5 /$ & $1.6 /$ & -1.3 & $-3.1 /$ & $-2.4 /$ & -1.8 & $-2.2 /$ & $-1.7 /$ & -1.7 & $-1.6 /$ & $-1.0 /$ & -1.1 \\
\hline $4 f^{3}\left({ }^{4} I\right) 5 d$ & ${ }^{5} I^{o}$ & 6 & 22047.8 & $-1.3 /$ & $2.7 /$ & -0.2 & $-1.9 /$ & $-1.4 /$ & -0.6 & $-0.9 /$ & $-0.5 /$ & -0.6 & $0.6 /$ & $0.9 /$ & 0.9 \\
\hline $4 f^{3}\left({ }^{4} I\right) 5 d$ & ${ }^{5} K^{o}$ & 9 & 22197.0 & $5.5 /$ & $8.6 /$ & 5.6 & $5.4 /$ & $5.0 /$ & 5.2 & $5.8 /$ & $5.4 /$ & 5.2 & $1.9 /$ & $1.6 /$ & 1.4 \\
\hline $4 f^{3}\left({ }^{4} I\right) 5 d$ & ${ }^{5} I^{o}$ & 7 & 22702.9 & $-1.0 /$ & $2.9 /$ & 0.2 & $-1.5 /$ & $-1.1 /$ & -0.3 & $-0.6 /$ & $-0.2 /$ & -5.8 & $0.6 /$ & $0.9 /$ & 0.9 \\
\hline $4 f^{3}\left({ }^{4} I\right) 5 d$ & ${ }^{5} H^{o}$ & 6 & 23819.3 & $-1.8 /$ & $1.9 /$ & -0.7 & $-2.3 /$ & $-1.8 /$ & -1.2 & $-1.5 /$ & $-1.1 /$ & -1.1 & $-1.2 /$ & $-0.7 /$ & -0.7 \\
\hline $4 f^{3}\left({ }^{4} I\right) 5 d$ & $o *$ & 7 & 24003.2 & & & & & & & & & & & & \\
\hline $4 f^{3}\left({ }^{4} I\right) 5 d$ & ${ }^{5} I^{o}$ & 8 & 24686.4 & $-1.3 /$ & $2.3 /$ & -0.2 & $-1.8 /$ & $-1.4 /$ & -0.6 & $-0.9 /$ & $-0.6 /$ & -0.6 & $1.4 /$ & $1.6 /$ & 1.6 \\
\hline $4 f^{3}\left({ }^{4} I\right) 5 d$ & $o *$ & 6 & 26503.2 & & & & & & & & & & & & \\
\hline $4 f^{3}\left({ }^{4} I\right) 5 d$ & ${ }^{3} K^{o}$ & 8 & 27391.4 & $-0.4 /$ & $3.0 /$ & 0.8 & $-0.8 /$ & $-0.4 /$ & 0.4 & $-0.1 /$ & $0.4 /$ & 0.5 & $-0.9 /$ & $-0.4 /$ & -0.3 \\
\hline $4 f^{3}\left({ }^{4} F\right) 5 d$ & $o *$ & 3 & 27569.8 & & & & & & & & & & & & \\
\hline \multirow[t]{2}{*}{$4 f^{3}\left({ }^{4} F\right) 5 d$} & ${ }^{5} H^{o}$ & 3 & 27788.2 & $-10.3 /$ & $-6.9 /$ & -8.9 & $-10.7 /$ & $-10.2 /$ & -9.3 & $-9.9 /$ & $-9.4 /$ & -9.2 & $-6.7 /$ & $-6.2 /$ & -6.1 \\
\hline & & 4 & 28745.3 & $-10.1 /$ & $-6.8 /$ & -8.8 & $-10.5 /$ & $-10.0 /$ & -9.2 & $-9.7 /$ & $-9.2 /$ & -9.1 & $-6.6 /$ & $-6.2 /$ & -6.1 \\
\hline $4 f^{3}\left({ }^{4} F\right) 5 d$ & $o *$ & 5 & 29397.3 & & & & & & & & & & & & \\
\hline \multirow[t]{3}{*}{$4 f^{3}\left({ }^{4} F\right) 5 d$} & ${ }^{5} H^{o}$ & 5 & 30232.3 & $-8.9 /$ & $-5.7 /$ & -7.6 & $-9.3 /$ & $-8.8 /$ & -8.0 & $-8.5 /$ & $-8.0 /$ & -7.9 & $-6.0 /$ & $-5.5 /$ & -5.4 \\
\hline & & 6 & 31394.6 & $-7.5 /$ & $-4.4 /$ & -6.1 & $-7.8 /$ & $-7.3 /$ & -6.5 & $-7.2 /$ & $-6.6 /$ & -6.4 & $-4.8 /$ & $-4.2 /$ & -5.0 \\
\hline & & 7 & 32832.6 & $-7.4 /$ & $-4.4 /$ & -6.1 & $-7.8 /$ & $-7.3 /$ & -6.5 & $-7.1 /$ & $-6.5 /$ & -6.4 & $-5.0 /$ & $-4.4 /$ & -4.3 \\
\hline
\end{tabular}




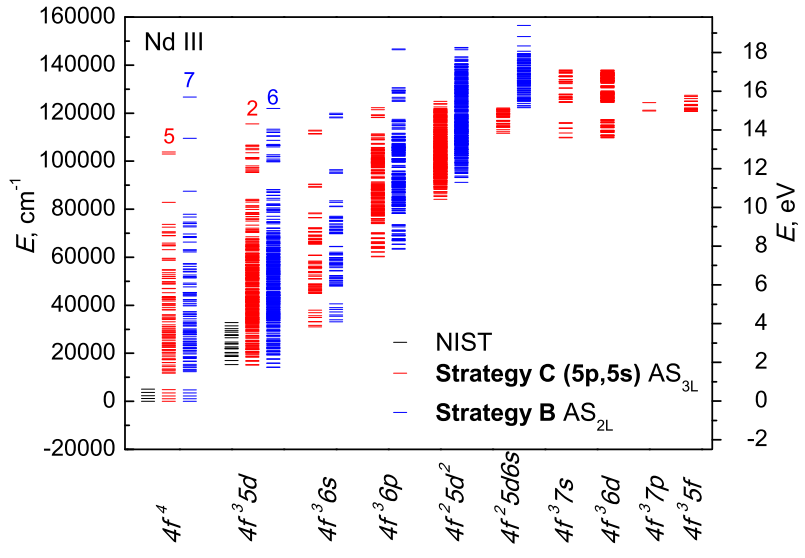

Figure 3. Energy levels for configurations of Nd III are compared with data of NIST. Black color is representing NIST data, next column of levels in red is our computed energy levels in Strategy C (5p, $\mathbf{5 s}) A S_{3 L}$, blue color data are based on Startegy B $A S_{2 L}$. Number on top of red and blue column is averaged disagreement in \% for levels of each configuration comparing with NIST database.

\section{3. $N d I V$}

Results of the energy levels of Strategies A and B are presented and compared with the NIST database in Table 6 . The best agreement with the NIST database is obtained for Strategy B with 5s. The energy levels are shown and compared with a few levels of configuration $4 f^{3}$ available in the NIST in Figure 4 . The averaged difference is $11 \%$ for Strategy B with $\mathbf{5 s}$ while it is $17 \%$ for Strategy A in active space $A S_{1 L}$.

For Nd IV ion, several experiments and analysis by semi-emperical methods have been performed. The emission spectrum produced by vacuum spark sources was observed in the vacuum ultraviolet on two normalincidence spectrographs. 550 lines have been identified as transitions from 85 (out of 107 possible) levels of $4 f^{2} 5 d$ to 37 (out of 41 possible) levels of $4 f^{3}$. The method and codes of Cowan were used to predict the spectral ranges of the strong transitions in the spectra $\mathrm{Nd}$ IV in the beginning of paper series (Wyart et al. 2006).

Later Wyart et al. (2007) used the same experiment to observe and classify 1426 lines. In total, 41 levels of $4 f^{3}$ configuration were reported. For deriving their energy levels with the diagonalization code RCG, the input Hartree-Fock radial integrals including relativistic corrections, treated as parameters (HFR parameters), were scaled according to earlier results on the neighbouring ions spectra. Altogether 111 odd parity and 121 even parity of configurations $4 f^{3}, 4 f^{2} 6 p, 5 p^{5} 4 f^{4}, 4 f^{2} 5 d$, $4 f^{2} 6 s$, and $5 p^{5} 4 f^{3} 5 d$ levels were established. Their op- timized values were calculated with the ELCALC code (Radziemski et al. 1970).

Then Wyart et al. (2008) performed a parametric fit of levels energies for $4 f^{3}$ configuration, previously obtained in the experiment (Wyart et al. 2007). Dzuba et al. (2003) did computation in the same way as for Nd III (see subsection 4.2). This included only 72 levels of configurations $4 f^{3}$ and $4 f^{2} 5 d$. In Table 7, the energy levels obtained applying Strategy B with 5s are compared with the experimental values by (Wyart et al. 2007) and semi-empirical values by Dzuba et al. (2003).

In addition to the energy levels, transition data can also be compared with experimental data and semiemperical calculations (Table 8). Our results on the transition wavelengths show good agreement with the experimental data by Wyart et al. (2007). As shown in Figure 5, the agreement in the wavelength is within 20 $\%$ for the most transitions.

We also confirmed a nice agreement in the transition probabilities. Table 8 and Figure 6 show transition probabilities for strongest transitions computed by Wyart et al. (2007). Our and their results agree within a factor of 2 . Note that semi-emperical calculations have uncertainties. Using the the same HFR method combined with parametric least-squares fits to the same experimental data with Wyart et al. (2007), Yoca \& Quinet (2014) have computed and presented transition probabilities (only with $\log g f \geq-1.0$ ), oscillator strengths and radiative lifetimes in bigger multiconfiguration expansions than Wyart et al. (2007). Their results are systematically different, and those by Yoca \& Quinet (2014) in fact show a slightly better agreement with ours as shown in the bottom panel of Figure 6.

\section{IMPACT TO THE OPACITIES}

We calculate bound-bound opacities using our results to study the impact of the accuracy in the atomic calculations. By following previous works on NS mergers (Kasen et al. 2013; Barnes \& Kasen 2013; Tanaka \& Hotokezaka 2013; Tanaka et al. 2014, 2018), we use the formalism of expansion opacity (Karp et al. 1977; Eastman \& Pinto 1993; Kasen et al. 2006):

$$
\kappa_{\exp }^{\mathrm{bb}}(\lambda)=\frac{1}{\rho c t} \sum_{l} \frac{\lambda_{l}}{\Delta \lambda}\left(1-e^{-\tau_{l}}\right) .
$$

Here, $\rho$ and $t$ represent density and time after the merger. The summation is taken over all the transitions in a wavelength bin $(\Delta \lambda)$, and $\lambda_{l}$ and $\tau_{l}$ are the transition wavelength and the Sobolev optical depth for each 
Table 5. Comparison of energy levels from present (Strategy C with $\mathbf{5 p}, \mathbf{5 s}$ ) and other theoretical computations with NIST database (in \%) of Nd III. States marked by subscript ${ }^{*}$ in term column are without term identification in the NIST database.

\begin{tabular}{|c|c|c|c|c|c|c|}
\hline \multirow[b]{2}{*}{ Config. } & \multirow[b]{2}{*}{ Term } & \multirow[b]{2}{*}{$J$} & \multirow[b]{2}{*}{ NIST } & \multirow{2}{*}{$\frac{\text { Present }}{\mathrm{AS}_{3 L}}$} & \multicolumn{2}{|c|}{ Dzuba et al. (2003) } \\
\hline & & & & & Cowan & RCI \\
\hline \multirow[t]{5}{*}{$4 f^{4}$} & ${ }^{5} I$ & 4 & 0.0 & 0 & 0 & 0 \\
\hline & & 5 & 1137.8 & $1073 / 5.7$ & $1137 / 0.1$ & $1162 /-2.1$ \\
\hline & & 6 & 2387.6 & $2264 / 5.2$ & $2397 /-0.4$ & $2471 /-3.5$ \\
\hline & & 7 & 3714.9 & $3543 / 4.6$ & $3743 /-0.8$ & $3898 /-4.9$ \\
\hline & & 8 & 5093.3 & $4885 / \quad 4.1$ & $5148 /-1.1$ & $5414 /-6.3$ \\
\hline \multirow[t]{3}{*}{$4 f^{3}\left({ }^{4} I\right) 5 d$} & ${ }^{5} K^{o}$ & 5 & 15262.2 & $15128 / 0.9$ & $14742 / \quad 3.4$ & $15357 /-0.6$ \\
\hline & & 6 & 16938.1 & $16721 / \quad 1.3$ & $16338 / 3.5$ & $17380 /-2.6$ \\
\hline & & 7 & 18656.3 & $18383 / 1.5$ & $18000 / 3.5$ & $19485 /-4.4$ \\
\hline $4 f^{3}\left({ }^{4} I\right) 5 d$ & ${ }^{5} I^{o}$ & 4 & 18883.7 & $18714 / \quad 0.9$ & $18467 / 2.2$ & $20284 /-7.4$ \\
\hline \multirow[t]{2}{*}{$4 f^{3}\left({ }^{4} I\right) 5 d$} & ${ }^{5} H^{o}$ & 3 & 19211.0 & $19753 /-2.8$ & $19427 /-1.1$ & $20946 /-9.0$ \\
\hline & & 4 & 20144.3 & $20465 /-1.6$ & $20189 /-0.2$ & $21926 /-8.8$ \\
\hline $4 f^{3}\left({ }^{4} I\right) 5 d$ & ${ }^{5} I^{o}$ & 5 & 20388.9 & $20208 / 0.9$ & $20006 / 1.9$ & $21254 /-4.2$ \\
\hline $4 f^{3}\left({ }^{4} I\right) 5 d$ & ${ }^{5} K^{o}$ & 8 & 20410.9 & $20105 / 1.5$ & $19725 / 3.4$ & $21666 /-6.1$ \\
\hline $4 f^{3}\left({ }^{4} I\right) 5 d$ & ${ }^{5} H^{o}$ & 5 & 21886.8 & $22119 /-1.1$ & $21866 / \quad 0.1$ & $22167 /-1.3$ \\
\hline $4 f^{3}\left({ }^{4} I\right) 5 d$ & ${ }^{5} I^{o}$ & 6 & 22047.8 & $21845 / \quad 0.9$ & $21672 / \quad 1.7$ & $22664 /-2.8$ \\
\hline $4 f^{3}\left({ }^{4} I\right) 5 d$ & ${ }^{5} K^{o}$ & 9 & 22197.0 & $21882 / \quad 1.4$ & $21503 / 3.1$ & $21919 / \quad 1.3$ \\
\hline $4 f^{3}\left({ }^{4} I\right) 5 d$ & ${ }^{5} I^{o}$ & 7 & 22702.9 & $22499 / \quad 0.9$ & $22244 / 2.0$ & $26537 /-16.9$ \\
\hline $4 f^{3}\left({ }^{4} I\right) 5 d$ & ${ }^{5} H^{o}$ & 6 & 23819.3 & $23992 /-0.7$ & $23733 / \quad 0.4$ & $24076 /-1.1$ \\
\hline $4 f^{3}\left({ }^{4} I\right) 5 d$ & $o *$ & 7 & 24003.2 & & & \\
\hline $4 f^{3}\left({ }^{4} I\right) 5 d$ & ${ }^{5} I^{o}$ & 8 & 24686.4 & $24301 / 1.6$ & $24158 / 2.1$ & $27396 /-11.0$ \\
\hline $4 f^{3}\left({ }^{4} I\right) 5 d$ & $o *$ & 6 & 26503.2 & & & \\
\hline $4 f^{3}\left({ }^{4} I\right) 5 d$ & ${ }^{3} K^{o}$ & 8 & 27391.4 & $27465 /-0.3$ & & \\
\hline $4 f^{3}\left({ }^{4} F\right) 5 d$ & $o *$ & 3 & 27569.8 & & & \\
\hline \multirow[t]{2}{*}{$4 f^{3}\left({ }^{4} F\right) 5 d$} & ${ }^{5} H^{o}$ & 3 & 27788.2 & $29494 /-6.1$ & $28824 /-3.7$ & \\
\hline & & 4 & 28745.3 & $30506 /-6.1$ & $29872 /-3.9$ & \\
\hline $4 f^{3}\left({ }^{4} F\right) 5 d$ & $o *$ & 5 & 29397.3 & & & \\
\hline \multirow[t]{3}{*}{$4 f^{3}\left({ }^{4} F\right) 5 d$} & ${ }^{5} H^{o}$ & 5 & 30232.3 & $31852 /-5.4$ & $31117 /-2.9$ & \\
\hline & & 6 & 31394.6 & $32961 /-5.0$ & $32054 /-2.1$ & \\
\hline & & 7 & 32832.6 & $34234 /-4.3$ & $33391 /-1.7$ & \\
\hline
\end{tabular}

transition. The Sobolev optical depth $\tau_{l}$ is expressed as

$$
\tau_{l}=\frac{\pi e^{2}}{m_{e} c} f_{l} n_{l} t \lambda_{l}=\frac{\pi e^{2}}{m_{e} c}\left(\frac{n \lambda_{l} t}{g_{0}}\right) g_{l} f_{l} \mathrm{e}^{-E_{l} / k T},
$$

where $g_{l}, E_{l}$, and $f_{l}$ are the statistical weight and the energy of the lower level of the transition and the oscillator strength of the transition, respectively. For the oscillator strength, we use results computed with the length (Babushkin) form. For the number density in the lower level of the transition $\left(n_{l}\right)$, the Boltzmann distribution is assumed, i.e., $n_{l}=\left(g_{l} / g_{0}\right) n \exp \left(-E_{l} / k T\right)$, where $g_{0}$ is the statistical weight for the ground level. The number density of each ion $n$ is calculated under the assumption of local thermodynamic equilibrium by using the Saha equation. In this paper, pure $\mathrm{Nd}$ gas is assumed. We use all the calculated transitions to evaluate the opacity without any selection based on the transition strengths, which was applied in full radiative transfer simulations (Tanaka et al. 2017).

We find that overall properties of opacities are not dramatically affected by the accuracies of the atomic 
Table 6. Comparison of energy levels with NIST database (in \%) of Nd IV by increase of the active space performed applying Strategies: A, B, and B with 5s. State marked by subscript ${ }^{*}$ in term column is without term identification in the NIST database.

\begin{tabular}{|c|c|c|c|c|c|c|c|c|c|c|c|c|}
\hline \multirow[b]{2}{*}{ Config. } & \multirow[b]{2}{*}{ Term } & \multirow[b]{2}{*}{$J$} & \multirow[b]{2}{*}{ NIST } & \multicolumn{3}{|c|}{ Strategy A } & \multicolumn{3}{|c|}{ Strategy B } & \multicolumn{3}{|c|}{ Strategy B (5s) } \\
\hline & & & & $\mathrm{AS}_{1 L} /$ & $\mathrm{AS}_{2 L} /$ & $\mathrm{AS}_{3 L}$ & $\mathrm{AS}_{1 L} /$ & $\mathrm{AS}_{2 L} /$ & $\mathrm{AS}_{3 L}$ & $\mathrm{AS}_{1 L} /$ & $\mathrm{AS}_{2 L} /$ & $\mathrm{AS}_{3 L}$ \\
\hline \multirow[t]{4}{*}{$4 f^{3}$} & ${ }^{4} I^{o}$ & $9 / 2$ & 0 & & & & & & & & & \\
\hline & & $11 / 2$ & [ 1880] & $6.8 /$ & $6.5 /$ & 6.4 & $6.9 /$ & $6.8 /$ & 6.7 & $7.2 /$ & $7.1 /$ & 7.0 \\
\hline & & $13 / 2$ & {$[3860]$} & $5.6 /$ & $5.4 /$ & 5.3 & $5.8 /$ & $5.8 /$ & 5.7 & $6.1 /$ & $6.1 /$ & 6.0 \\
\hline & & $15 / 2$ & {$[5910]$} & $4.7 /$ & $4.6 /$ & 4.6 & $4.9 /$ & $5.0 /$ & 5.0 & $5.2 /$ & $5.3 /$ & 5.3 \\
\hline \multirow[t]{2}{*}{$4 f^{3}$} & ${ }^{4} F^{o}$ & $3 / 2$ & [11290] & $-27.8 /$ & $-27.0 /$ & -26.6 & $-20.3 /$ & $-19.2 /$ & -18.6 & $-17.6 /$ & $-16.4 /$ & -15.8 \\
\hline & & $5 / 2$ & [12320] & $-24.8 /$ & $-24.2 /$ & -23.8 & $-17.9 /$ & $-16.9 /$ & -16.4 & $-15.3 /$ & $-14.4 /$ & -13.8 \\
\hline $4 f^{3}$ & ${ }^{2} H 2^{\circ}$ & $9 / 2$ & {$[12470]$} & $-14.7 /$ & $-12.5 /$ & -11.3 & $-12.8 /$ & $-10.4 /$ & -9.4 & $-12.0 /$ & $-9.5 /$ & -8.5 \\
\hline $4 f^{3}$ & ${ }^{4} F^{o}$ & $7 / 2$ & [13280] & $-22.3 /$ & $-21.6 /$ & -21.2 & $-16.1 /$ & $-15.2 /$ & -14.6 & $-13.7 /$ & $-12.8 /$ & -12.3 \\
\hline $4 f^{3}$ & ${ }^{4} S^{o}$ & $3 / 2$ & [13370] & $-20.2 /$ & $-16.8 /$ & -16.5 & $-15.6 /$ & $-12.1 /$ & -11.6 & $-13.3 /$ & $-9.8 /$ & -9.3 \\
\hline $4 f^{3}$ & ${ }^{4} F^{o}$ & $9 / 2$ & {$[14570]$} & $-18.3 /$ & $-17.6 /$ & -17.1 & $-13.2 /$ & $-12.1 /$ & -11.6 & $-11.3 /$ & $-10.2 /$ & -9.7 \\
\hline $4 f^{3}$ & ${ }^{2} H 2^{\circ}$ & $11 / 2$ & [15800] & $-9.6 /$ & $-7.8 /$ & -6.8 & $-8.4 /$ & $-6.3 /$ & -5.5 & $-7.8 /$ & $-5.7 /$ & -4.9 \\
\hline $4 f^{3}$ & ${ }^{4} G^{o}$ & $5 / 2$ & [16980] & $-29.6 /$ & $-28.1 /$ & -27.8 & $-21.7 /$ & $-20.2 /$ & -19.6 & $-18.6 /$ & $-17.1 /$ & -16.5 \\
\hline $4 f^{3}$ & $o *$ & $7 / 2$ & [17100] & & & & & & & & & \\
\hline \multirow[t]{2}{*}{$4 f^{3}$} & ${ }^{4} G^{o}$ & $7 / 2$ & [18890] & $-23.6 /$ & $-22.3 /$ & -22.0 & $-16.9 /$ & $-15.5 /$ & -14.9 & $-14.3 /$ & $-12.9 /$ & -12.3 \\
\hline & & $9 / 2$ & [19290] & $-16.6 /$ & $-27.0 /$ & -26.7 & $-22.1 /$ & $-20.6 /$ & -20.0 & $-19.7 /$ & $-18.3 /$ & -17.7 \\
\hline $4 f^{3}$ & ${ }^{2} K^{o}$ & $13 / 2$ & [19440] & $-17.7 /$ & $-15.0 /$ & -14.2 & $-15.1 /$ & $-12.2 /$ & -11.4 & $-13.9 /$ & $-11.1 /$ & -10.3 \\
\hline $4 f^{3}$ & ${ }^{4} G^{o}$ & $11 / 2$ & [21280] & $-22.2 /$ & $-21.0 /$ & -20.8 & $-15.9 /$ & $-14.6 /$ & -14.2 & $-13.4 /$ & $-12.2 /$ & -11.7 \\
\hline $4 f^{3}$ & ${ }^{2} K^{o}$ & $15 / 2$ & [21430] & $-16.1 /$ & $-13.7 /$ & -12.9 & $-13.6 /$ & $-11.0 /$ & -10.3 & $-12.5 /$ & $-9.9 /$ & -9.2 \\
\hline
\end{tabular}

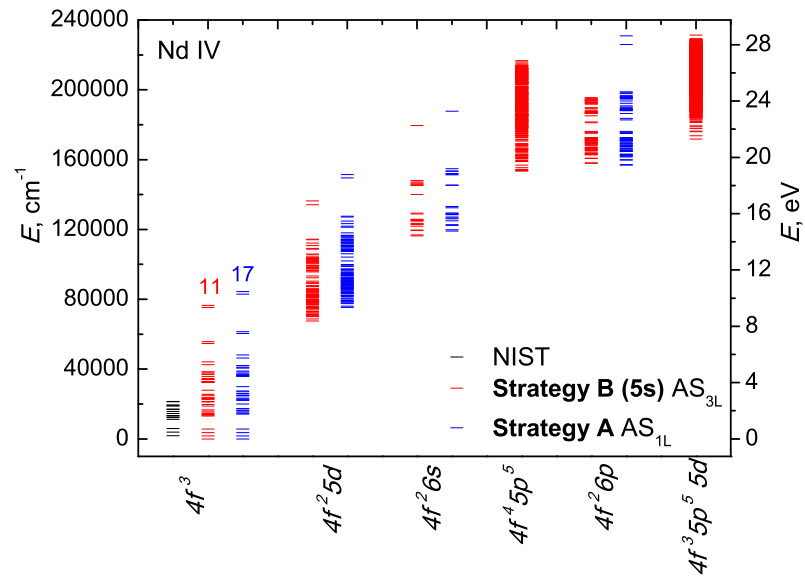

Figure 4. Energy levels for configurations of Nd IV are compared with data of NIST. Black color is representing NIST data, next column of levels in red is our computed energy levels in Strategy B (5s) $A S_{3 L}$, blue color data are based on Startegy A $A S_{1 L}$. Number on top of red and blue column is averaged disagreement in \% for levels of each configuration comparing with NIST database.

calculations. Left panels in figure 7 shows the expansion opacities calculated by using transition data of $\mathrm{Nd}$

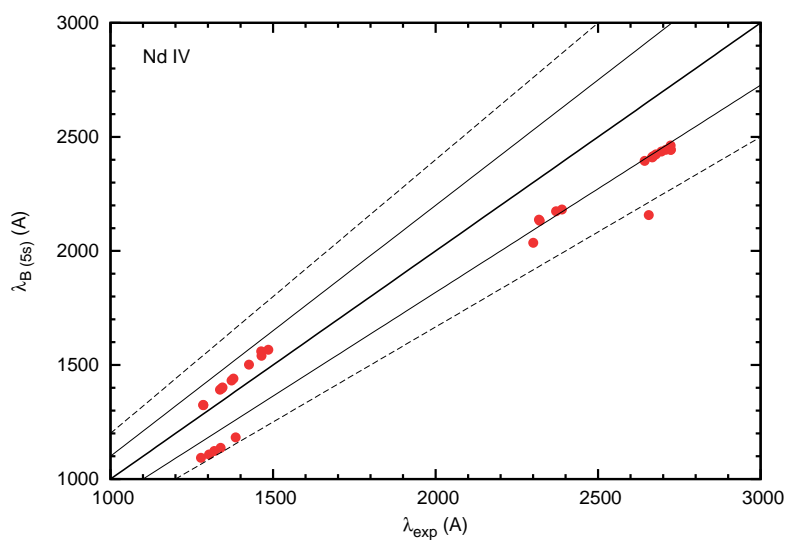

Figure 5. Comparison of transition wavelengths for Nd IV between our results from Strategy B with 5s and experimental data by Wyart et al. (2007). The thick line corresponds to the perfect agreement while thin solid and dashed lines correspond to $10 \%$ and $20 \%$ deviation.

II, Nd III, and Nd IV. The temperature is assumed to be $5000 \mathrm{~K}, 10000 \mathrm{~K}$, and $15000 \mathrm{~K}$ for Nd II, Nd III, and Nd IV, respectively. The density is $1 \times 10^{-13} \mathrm{~g} \mathrm{~cm}^{-3}$ and time after the merger is set to be 1 day. Overall opacity values and wavelength dependence are quite 
Table 7. Comparison of energy levels from present (Strategy B with 5s) and other theoretical computations with NIST database (in \%) and with the experiment Wyart et al. (2007) (in \%) of Nd IV. State marked by subscript ${ }^{*}$ in term column is without term identification in the NIST database.

\begin{tabular}{|c|c|c|c|c|c|c|c|}
\hline \multirow[b]{2}{*}{ Config. } & \multirow[b]{2}{*}{ Term } & \multirow[b]{2}{*}{$J$} & \multirow[b]{2}{*}{ NIST } & \multirow[b]{2}{*}{ Exp. } & \multirow{2}{*}{$\frac{\text { Present }}{\mathrm{AS}_{3 L}}$} & \multicolumn{2}{|c|}{ Dzuba et al. (2003) } \\
\hline & & & & & & Cowan & $\mathrm{RCI}$ \\
\hline \multirow[t]{4}{*}{$4 f^{3}$} & ${ }^{4} I^{o}$ & $9 / 2$ & 0 & 0 & 0 & 0 & 0 \\
\hline & & $11 / 2$ & [ 1880] & 1897.11 & $1749 /$ & $0.1 / \quad 1.0$ & $1945 /-3.5 /-2.5$ \\
\hline & & $13 / 2$ & {$[3860]$} & 3907.43 & $3627 /$ & $3890 /-0.8 /$ & $4049 /-4.9 /-3.6$ \\
\hline & & $15 / 2$ & [ 5910] & 5988.51 & $5596 /$ & $5989 /-1.3 /$ & $6267 /-6.0 /-4.7$ \\
\hline \multirow[t]{2}{*}{$4 f^{3}$} & ${ }^{4} F^{o}$ & $3 / 2$ & [11290] & 11698.49 & $13076 /-15.8 /-11.8$ & $13294 /-17.8 /-13.6$ & $12490 /-10.6 /-6.8$ \\
\hline & & $5 / 2$ & {$[12320]$} & 12747.94 & $14022 /-13.8 /-10.0$ & $14333 /-16.3 /-12.4$ & $13545 /-9.9 /-6.3$ \\
\hline $4 f^{3}$ & ${ }^{2} H 2^{o}$ & $9 / 2$ & {$[12470]$} & 12800.29 & $13536 /-8.5 /-5.8$ & $13272 /-6.4 /-3.7$ & $14522 /-16.5 /-13.5$ \\
\hline $4 f^{3}$ & ${ }^{4} F^{o}$ & $7 / 2$ & {$[13280]$} & 13719.82 & $14911 /-12.3 /-8.7$ & $15249 /-14.8 /-11.1$ & $14622 /-10.1 /-6.6$ \\
\hline $4 f^{3}$ & ${ }^{4} S^{\circ}$ & $3 / 2$ & [13370] & 13792.49 & $14617 /-9.3 /-6.0$ & $15153 /-13.3 /-9.9$ & $14452 /-8.1 /-4.8$ \\
\hline $4 f^{3}$ & ${ }^{4} F^{o}$ & $9 / 2$ & {$[14570]$} & 14994.87 & $15979 /-9.7 /-6.6$ & $16334 /-12.1 /-8.9$ & $16183 /-11.1 /-7.9$ \\
\hline $4 f^{3}$ & ${ }^{2} H 2^{\circ}$ & $11 / 2$ & {$[15800]$} & 16161.53 & $16581 /-4.9 /-2.6$ & $16456 /-4.2 /-1.8$ & $18142 /-14.8 /-12.3$ \\
\hline $4 f^{3}$ & ${ }^{4} G^{o}$ & $5 / 2$ & [16980] & 17707.17 & $19780 /-16.5 /-11.7$ & & \\
\hline $4 f^{3}$ & $o *$ & $7 / 2$ & {$[17100]$} & 17655.11 & & & \\
\hline \multirow[t]{2}{*}{$4 f^{3}$} & ${ }^{4} G^{o}$ & $7 / 2$ & {$[18890]$} & 19540.80 & $21218 /-12.3 /-8.6$ & & \\
\hline & & $9 / 2$ & [19290] & 19969.79 & $22709 /-17.7 /-13.7$ & & \\
\hline $4 f^{3}$ & ${ }^{2} K^{o}$ & $13 / 2$ & [19440] & 20005.22 & $21445 /-10.3 /-7.2$ & & \\
\hline $4 f^{3}$ & ${ }^{4} G^{o}$ & $11 / 2$ & {$[21280]$} & 22047.39 & $23768 /-11.7 /-7.8$ & & \\
\hline $4 f^{3}$ & ${ }^{2} K^{o}$ & $15 / 2$ & {$[21430]$} & 22043.77 & $23398 /-9.2 /-6.1$ & & \\
\hline
\end{tabular}

similar for different atomic calculations. The red lines show the best results in this paper while blue lines show the previous results used by Tanaka et al. (2018).

The behaviors of the opacity are also similar for different temperature. Right panels show the Planck mean opacities calculated for different temperatures by keeping the density and time to be the same. The Planck mean opacity from different atomic calculations agree with each other within a factor of 1.5. Since the timescale of the kilonova emission scales as $\kappa^{0.5}$ (Rosswog 2015; Tanaka 2016; Fernández \& Metzger 2016; Metzger 2017), this level of differences does not significantly affect the timescale of kilonova (smaller than $\sim 20 \%$ ) compared with those expected from differences in temperature and abundances.

With a close look, however, the wavelength dependent opacities show some differences. The most notable difference is the feature around $4000 \AA$ in the case of Nd II. The new GRASP2K calculations with a better accuracy show a bump while the old GRASP2K calculations and HULLAC calculations do not, making a difference in the opacity by a factor of 2 . Interestingly, Kasen et al. (2013) also showed that this part of the opacity is af- fected by the optimization in the atomic calculations: the peak is located near $5000 \AA$ in their opt1 case while the peak is weaker in their opt 2 and opt 3 cases. The expansion opacities presented by Fontes et al. (2017) also show a peak around $4000 \AA$, which is close to our new results.

We find that the difference between our new and previous opacities is caused by the lower energy levels of $4 f^{3} 5 d^{2}$ and $4 f^{3} 5 d 6 s$ configurations in our new calculations (Figure 1). Figure 8 shows the number of strong transitions which fulfill $g_{l} f_{l} \exp \left(-E_{l} / k T\right)>10^{-5}$ at $T=5000 \mathrm{~K}$. The numbers of transition are separated according to the lower level configuration. The number of strong transition from the levels of $4 f^{3} 5 d^{2}$ and $4 f^{3} 5 d 6 s$ configuration is enhanced around $4500 \AA$ in our new calculations (Strategy C $A S_{2 L}$ ). Since the energy of these configurations are overestimated in our previous calculations (Strategy A $A S_{2 L}$, Figure 1), the bump structure in the new calculations seems more realistic. This demonstrates the importance of accurate calculations for lower energy levels to predict spectra of kilonova. 
Table 8. Computed transitions data of Nd IV in the Strategies B with $\mathbf{5 s}$ at $A S_{3 L}$ and compared with the experimental wavelength $\lambda_{\exp }$ (in $\AA$ ) and computed transitions probabilities $A_{S E}$ (in s ${ }^{-1}$ ) by Wyart et al. (2007).

\begin{tabular}{|c|c|c|c|c|c|c|c|c|c|c|}
\hline \multirow[b]{2}{*}{ Upper } & \multirow{2}{*}{\multicolumn{3}{|c|}{ Lower }} & \multicolumn{4}{|c|}{ Strategies B with 5s } & \multicolumn{2}{|c|}{ Wyart et al. (2007) } & \multirow{2}{*}{$\begin{array}{r}\text { Yoca \& Quinet (2014) } \\
\qquad A_{S E}\end{array}$} \\
\hline & & & & $A_{1}$ & $A_{\mathrm{v}}$ & $d T$ & $\lambda$ & $A_{S E}$ & $\lambda_{\exp }$ & \\
\hline $4 f^{2}\left({ }^{3} H\right) 6 p{ }^{4} I$ & 4.5 & $4 f^{2}\left({ }^{3} H\right) 5 d^{2} H$ & 4.5 & $5.26 \mathrm{E}+8$ & $4.40 \mathrm{E}+8$ & 0.16 & $1107.72(15.0)$ & $4.04 \mathrm{E}+8$ & 1303.32 & $3.89 \mathrm{E}+8$ \\
\hline $4 f^{2}\left({ }^{1} I\right) 5 d^{2} K$ & 7.5 & $4 f^{32} L$ & 8.5 & $9.32 \mathrm{E}+7$ & $1.24 \mathrm{E}+8$ & 0.25 & $1540.16(-5.1)$ & $1.64 \mathrm{E}+8$ & 1464.73 & $1.47 \mathrm{E}+8$ \\
\hline $4 f^{2}\left({ }^{3} H\right) 6 p{ }^{4} I$ & 5.5 & $4 f^{2}\left({ }^{3} H\right) 6 s{ }^{4} H$ & 5.5 & $2.04 \mathrm{E}+8$ & $1.91 \mathrm{E}+8$ & 0.06 & $2412.27(9.5)$ & $1.96 \mathrm{E}+8$ & 2666.70 & $1.94 \mathrm{E}+8$ \\
\hline $4 f^{2}\left({ }^{3} H\right) 6 p{ }^{4} I$ & 4.5 & $4 f^{2}\left({ }^{3} H\right) 6 s{ }^{4} H$ & 3.5 & $1.92 \mathrm{E}+8$ & $1.61 \mathrm{E}+8$ & 0.16 & $2410.31(9.6)$ & $1.97 \mathrm{E}+8$ & 2666.70 & $1.96 \mathrm{E}+8$ \\
\hline $4 f^{2}\left({ }^{1} G\right) 5 d^{2} I$ & 6.5 & $4 f^{32} K$ & 7.5 & $6.85 \mathrm{E}+7$ & $8.99 \mathrm{E}+7$ & 0.24 & $1559.66(-6.6)$ & $1.28 \mathrm{E}+8$ & 1463.34 & $1.14 \mathrm{E}+8$ \\
\hline $4 f^{2}\left({ }^{3} F\right) 5 d{ }^{4} H$ & 5.5 & $4 f^{3}{ }^{4} I$ & 6.5 & $3.78 \mathrm{E}+7$ & $5.67 \mathrm{E}+7$ & 0.33 & $1324.01(-3.0)$ & $8.92 \mathrm{E}+7$ & 1285.61 & $6.48 \mathrm{E}+7$ \\
\hline $4 f^{2}\left({ }^{3} F\right) 5 d{ }^{4} H$ & 4.5 & $4 f^{3}{ }^{4} I$ & 5.5 & $4.11 \mathrm{E}+7$ & $6.19 \mathrm{E}+7$ & 0.34 & $1325.17(-3.1)$ & $1.04 \mathrm{E}+8$ & 1285.38 & $7.55 \mathrm{E}+7$ \\
\hline $4 f^{2}\left({ }^{3} H\right) 5 d^{4} H$ & 3.5 & $4 f^{3}{ }^{4} I$ & 4.5 & $7.27 \mathrm{E}+7$ & $1.03 \mathrm{E}+8$ & 0.29 & $1401.4(-4.2)$ & $1.29 \mathrm{E}+8$ & 1344.74 & $1.17 \mathrm{E}+8$ \\
\hline $4 f^{2}\left({ }^{3} H\right) 6 p{ }^{4} I$ & 4.5 & $4 f^{2}\left({ }^{3} H\right) 5 d{ }^{4} K$ & 5.5 & $1.01 \mathrm{E}+9$ & $8.05 \mathrm{E}+8$ & 0.20 & $1123.17(14.9)$ & $7.61 \mathrm{E}+8$ & 1319.25 & $7.29 \mathrm{E}+8$ \\
\hline $4 f^{2}\left({ }^{3} H\right) 6 p{ }^{4} G$ & 5.5 & $4 f^{2}\left({ }^{3} H\right) 6 s{ }^{4} H$ & 6.5 & $1.55 \mathrm{E}+8$ & $1.65 \mathrm{E}+8$ & 0.06 & $2157.57(18.8)$ & $2.17 \mathrm{E}+8$ & 2656.02 & $2.16 \mathrm{E}+8$ \\
\hline $4 f^{2}\left({ }^{1} I\right) 6 p{ }^{2} I$ & 5.5 & $4 f^{2}\left({ }^{1} I\right) 6 s{ }^{2} I$ & 5.5 & $2.30 \mathrm{E}+8$ & $2.17 \mathrm{E}+8$ & 0.06 & $2443.54(10.3)$ & $1.70 \mathrm{E}+8$ & 2723.51 & $1.86 \mathrm{E}+8$ \\
\hline $4 f^{2}\left({ }^{3} H\right) 6 p{ }^{4} H$ & 4.5 & $4 f^{2}\left({ }^{3} H\right) 6 s{ }^{4} H$ & 5.5 & $2.02 \mathrm{E}+8$ & $2.07 \mathrm{E}+8$ & 0.02 & $2416.08(9.5)$ & $2.00 \mathrm{E}+8$ & 2670.03 & $2.04 \mathrm{E}+8$ \\
\hline $4 f^{2}\left({ }^{3} H\right) 6 p{ }^{4} H$ & 3.5 & $4 f^{2}\left({ }^{3} H\right) 6 s{ }^{4} H$ & 4.5 & $1.95 \mathrm{E}+8$ & $2.00 \mathrm{E}+8$ & 0.02 & $2423.25(9.5)$ & $1.99 \mathrm{E}+8$ & 2678.00 & \\
\hline $4 f^{2}\left({ }^{3} H\right) 5 d{ }^{4} H$ & 5.5 & $4 f^{34} I$ & 6.5 & $7.27 \mathrm{E}+7$ & $1.04 \mathrm{E}+8$ & 0.30 & $1391.9(-4.1)$ & $1.29 \mathrm{E}+8$ & 1336.98 & $1.00 \mathrm{E}+8$ \\
\hline $4 f^{2}\left({ }^{3} H\right) 5 d^{4} I$ & 7.5 & $4 f^{3}{ }^{4} I$ & 7.5 & $4.69 \mathrm{E}+7$ & $8.18 \mathrm{E}+7$ & 0.43 & $1432.37(-4.4)$ & $9.05 \mathrm{E}+7$ & 1372.20 & $7.44 \mathrm{E}+7$ \\
\hline $4 f^{2}\left({ }^{1} I\right) 6 p^{2} I$ & 5.5 & $4 f^{2}\left({ }^{1} I\right) 6 s^{2} I$ & 6.5 & $1.16 \mathrm{E}+8$ & $1.20 \mathrm{E}+8$ & 0.03 & $2443.59(10.3)$ & $1.17 \mathrm{E}+8$ & 2723.51 & $1.22 \mathrm{E}+8$ \\
\hline $4 f^{2}\left({ }^{3} H\right) 6 p{ }^{4} H$ & 4.5 & $4 f^{2}\left({ }^{3} H\right) 6 s{ }^{2} H$ & 4.5 & $1.38 \mathrm{E}+8$ & $1.30 \mathrm{E}+8$ & 0.05 & $2436.05(9.6)$ & $1.27 \mathrm{E}+8$ & 2694.71 & \\
\hline $4 f^{2}\left({ }^{3} H\right) 5 d{ }^{4} H$ & 4.5 & $4 f^{3}{ }^{4} I$ & 5.5 & $7.02 \mathrm{E}+7$ & $9.97 \mathrm{E}+7$ & 0.30 & $1397.63(-4.1)$ & $1.24 \mathrm{E}+8$ & 1342.01 & $1.10 \mathrm{E}+8$ \\
\hline $4 f^{2}\left({ }^{3} H\right) 6 p^{2} I$ & 6.5 & $4 f^{2}\left({ }^{3} H\right) 6 s{ }^{2} H$ & 5.5 & $3.29 \mathrm{E}+8$ & $2.88 \mathrm{E}+8$ & 0.13 & $2174(8.3)$ & $2.84 \mathrm{E}+8$ & 2370.51 & $2.94 \mathrm{E}+8$ \\
\hline $4 f^{2}\left({ }^{3} F\right) 6 p^{4} D$ & 3.5 & $4 f^{2}\left({ }^{3} F\right) 6 s{ }^{4} F$ & 4.5 & $2.86 \mathrm{E}+8$ & $2.88 \mathrm{E}+8$ & 0.01 & 2394.67( 9.4$)$ & $2.89 \mathrm{E}+8$ & 2643.03 & $2.93 \mathrm{E}+8$ \\
\hline $4 f^{2}\left({ }^{1} I\right) 6 p^{2} K$ & 6.5 & $4 f^{2}\left({ }^{1} I\right) 6 s^{2} I$ & 6.5 & $7.68 \mathrm{E}+7$ & $7.75 \mathrm{E}+7$ & 0.01 & $2181.33(8.7)$ & $8.11 \mathrm{E}+7$ & 2388.79 & $1.26 \mathrm{E}+8$ \\
\hline $4 f^{2}\left({ }^{3} H\right) 6 p^{4} I$ & 7.5 & $4 f^{2}\left({ }^{3} H\right) 5 d{ }^{4} K$ & 8.5 & $1.34 \mathrm{E}+9$ & $1.07 \mathrm{E}+9$ & 0.20 & $1093.02(14.5)$ & $6.16 \mathrm{E}+8$ & 1278.41 & $6.63 \mathrm{E}+8$ \\
\hline $4 f^{2}\left({ }^{3} H\right) 6 p^{2} I$ & 6.5 & $4 f^{2}\left({ }^{3} H\right) 5 d^{2} K$ & 7.5 & $7.92 \mathrm{E}+8$ & $6.02 \mathrm{E}+8$ & 0.24 & $1182.67(14.6)$ & $4.37 \mathrm{E}+8$ & 1385.21 & $4.89 \mathrm{E}+8$ \\
\hline $4 f^{2}\left({ }^{3} H\right) 6 p{ }^{4} I$ & 6.5 & $4 f^{2}\left({ }^{3} H\right) 6 s{ }^{4} H$ & 5.5 & $4.95 \mathrm{E}+8$ & $4.43 \mathrm{E}+8$ & 0.10 & $2132.31(8.1)$ & $3.65 \mathrm{E}+8$ & 2320.43 & $4.09 \mathrm{E}+8$ \\
\hline $4 f^{2}\left({ }^{3} F\right) 6 p{ }^{4} G$ & 5.5 & $4 f^{2}\left({ }^{3} F\right) 6 s{ }^{4} F$ & 4.5 & $5.00 \mathrm{E}+8$ & $4.56 \mathrm{E}+8$ & 0.09 & $2137.32(7.8)$ & $4.15 \mathrm{E}+8$ & 2318.07 & $3.98 \mathrm{E}+8$ \\
\hline $4 f^{2}\left({ }^{3} H\right) 6 p^{2} I$ & 5.5 & $4 f^{2}\left({ }^{3} H\right) 6 s{ }^{4} H$ & 4.5 & $1.90 \mathrm{E}+7$ & $1.56 \mathrm{E}+7$ & 0.18 & $2035.66(11.5)$ & $3.96 \mathrm{E}+8$ & 2300.68 & $4.16 \mathrm{E}+8$ \\
\hline $4 f^{2}\left({ }^{3} H\right) 6 p{ }^{4} H$ & 5.5 & $4 f^{2}\left({ }^{3} H\right) 5 d{ }^{4} H$ & 5.5 & $2.85 \mathrm{E}+8$ & $2.39 \mathrm{E}+8$ & 0.16 & $1136.61(15.1)$ & $1.54 \mathrm{E}+8$ & 1338.62 & $1.03 \mathrm{E}+8$ \\
\hline $4 f^{2}\left({ }^{1} I\right) 5 d^{2} K$ & 6.5 & $4 f^{32} L$ & 7.5 & $6.41 \mathrm{E}+7$ & $8.42 \mathrm{E}+7$ & 0.24 & $1501.86(-5.3)$ & $1.27 \mathrm{E}+8$ & 1426.05 & $1.14 \mathrm{E}+8$ \\
\hline $4 f^{2}\left({ }^{3} H\right) 6 p{ }^{4} I$ & 4.5 & $4 f^{2}\left({ }^{3} H\right) 6 s{ }^{4} H$ & 4.5 & $1.78 \mathrm{E}+8$ & $1.65 \mathrm{E}+8$ & 0.07 & $2443.44(9.8)$ & $1.71 \mathrm{E}+8$ & 2708.43 & $1.70 \mathrm{E}+8$ \\
\hline $4 f^{2}\left({ }^{3} H\right) 6 p{ }^{4} H$ & 5.5 & $4 f^{2}\left({ }^{3} H\right) 6 s^{2} H$ & 5.5 & $1.16 \mathrm{E}+8$ & $1.10 \mathrm{E}+8$ & 0.06 & $2462.26(9.6)$ & $1.18 \mathrm{E}+8$ & 2723.34 & $1.23 \mathrm{E}+8$ \\
\hline $4 f^{2}\left({ }^{3} H\right) 5 d^{4} I$ & 6.5 & $4 f^{3}{ }^{4} I$ & 6.5 & $4.40 \mathrm{E}+7$ & $7.69 \mathrm{E}+7$ & 0.43 & $1440.32(-4.5)$ & $8.54 \mathrm{E}+7$ & 1378.09 & $6.95 \mathrm{E}+7$ \\
\hline $4 f^{2}\left({ }^{1} I\right) 5 d^{2} I$ & 6.5 & $4 f^{32} K$ & 7.5 & $3.75 \mathrm{E}+7$ & $4.99 \mathrm{E}+7$ & 0.25 & $1566.59(-5.4)$ & $7.14 \mathrm{E}+7$ & 1485.64 & $6.43 \mathrm{E}+7$ \\
\hline
\end{tabular}

Figure 9 shows the cumulative number of states (CNS) for $4 f^{3} 5 d^{2}$ and $4 f^{3} 5 d 6 s$ configurations as a function of excitation energies. It is noted that the number of states takes the statistical-weight (degeneracy) of each level, i. e. $2 J+1$, into account. The CNS for the whole energy levels obtained by calculations with GRASP2K and HULLAC is compared in the figure. The CNS of the new calculations gets rising at a lower energy and has larger values than those of the previous calculations with
GRASP2K and HULLAC, indicating that the larger number of states falls into the lower energy region with the new calculations. Since the Boltzmann distribution is assumed for the number density in the lower levels of transitions, it is predicted that the number of strong transitions from the levels of $4 f^{3} 5 d^{2}$ and $4 f^{3} 5 d 6 s$ configurations becomes larger with the new calculations as depicted in Fig. 8. This is more remarkable for $4 f^{3} 5 d 6 \mathrm{~s}$ configuration as in the CNS. The CNS of the new cal- 

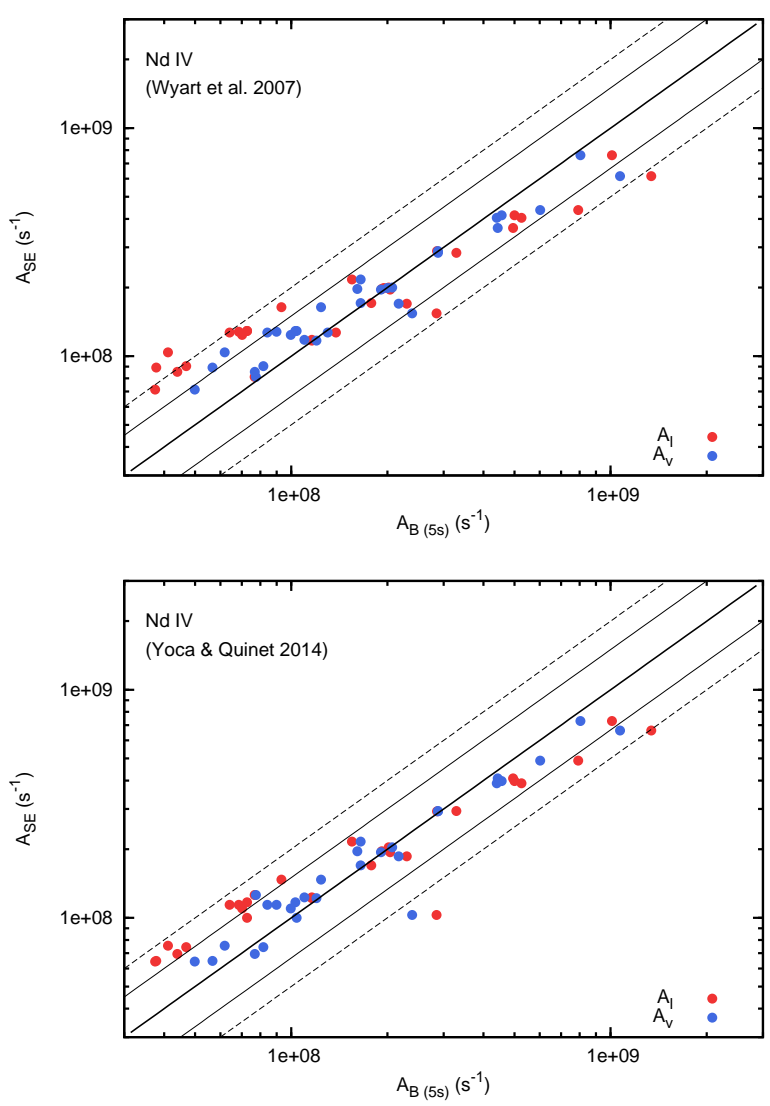

Figure 6. Comparison of transition probability for Nd IV. The top and bottom panels show comparison between our results from Strategy B with $\mathbf{5 s}$ and semi-emperical results by Wyart et al. (2007) and by Yoca \& Quinet (2014), respectively. The thick line corresponds to the perfect agreement while thin solid and dashed lines correspond to the deviation by a factor of 1.5 and 2.0, respectively. Red and blue points show the values calculated with the length (Babushkin) and velocity (Coulomb) forms, respectively.

culations is compared also with those of NIST and the semi-empirical results by Wyart (2010). Overall agreement is good at low energies convincing accuracy of our new calculations. Only exception is the semi-empirical results for $4 f^{3} 5 d^{2}$ configuration which overshoot significantly at high energies. Reasons of this discrepancy are yet to be investigated.

Another notable difference is a feature around $1000 \AA$ : the opacities in our new calculations are suppressed. This is due to the inclusion of highly excited energy levels in the previous calculations (both GRASP2K and HULLAC). Therefore, the opacities in the ultraviolet wavelengths depends on the choice of the configurations included in the calculations. However, if configurations with sufficiently high energy $(E \sim 10-15 \mathrm{eV})$ are in- cluded, such a difference appears only in the far ultraviolet wavelength, and thus, does not affect observable features.

\section{SUMMARY}

We presented extensive atomic calculations of neodymium and studied impact of accuracies in the calculations to the astrophysical opacities. The extended search of electron correlation effect inclusion strategies is presented in this work for the three Nd ions (Nd II, III and IV). In total, 6 000, 1 453, 1533 levels are presented for Nd II, Nd III and Nd IV respectively, and E1 type transitions between these levels were computed. Exclusive accuracy is achieved for atomic energy spectra results. Compared with NIST database, the averaged relative differences are $10 \%, 3 \%$, and $11 \%$ for Nd II, Nd III, and Nd IV, respectively.

Using our new results, we calculated expansion opacities used in radiative transfer simulations for kilonova, radioactively-powered EM emission from NS merger. We found that the overall opacities values and their wavelength dependence are not very sensitive to the accuracies of the calculations. The Planck mean opacities from our previous and new atomic calculations agree within a factor of 1.5. This confirms the validity of previous studies on kilonova.

However, some wavelength dependent features are affected by the accuracy of atomic calculations. In particular, the low-lying energy levels $(E<2-3 \mathrm{eV})$ can affect the opacities and even produce a bump in a certain wavelength range. Our results highlight importance of accurate atomic calculations for low-lying energy levels to accurately predict the spectra of kilonova.

This research was funded by a grant (No. S-LJB-18-1) from the Research Council of Lithuania. This research was also supported by JSPS Bilateral Joint Research Project. Computations presented in this paper were performed at the High Performance Computing Center "HPC Sauletekis" of the Faculty of Physics at Vilnius University and with Cray XC30 and XC50 at Center for Computational Astrophysics, National Astronomical Observatory of Japan. DK is grateful to the support by NINS program of Promoting Research by Networking among Institutions (Grant Number 01411702). MT is supported by the NINS program for cross-disciplinary science study, Inoue Science Research Award from Inoue Foundation for Science, and the Grant-in-Aid for Scientific Research from JSPS (15H02075, 16H02183) and MEXT (17H06363). 

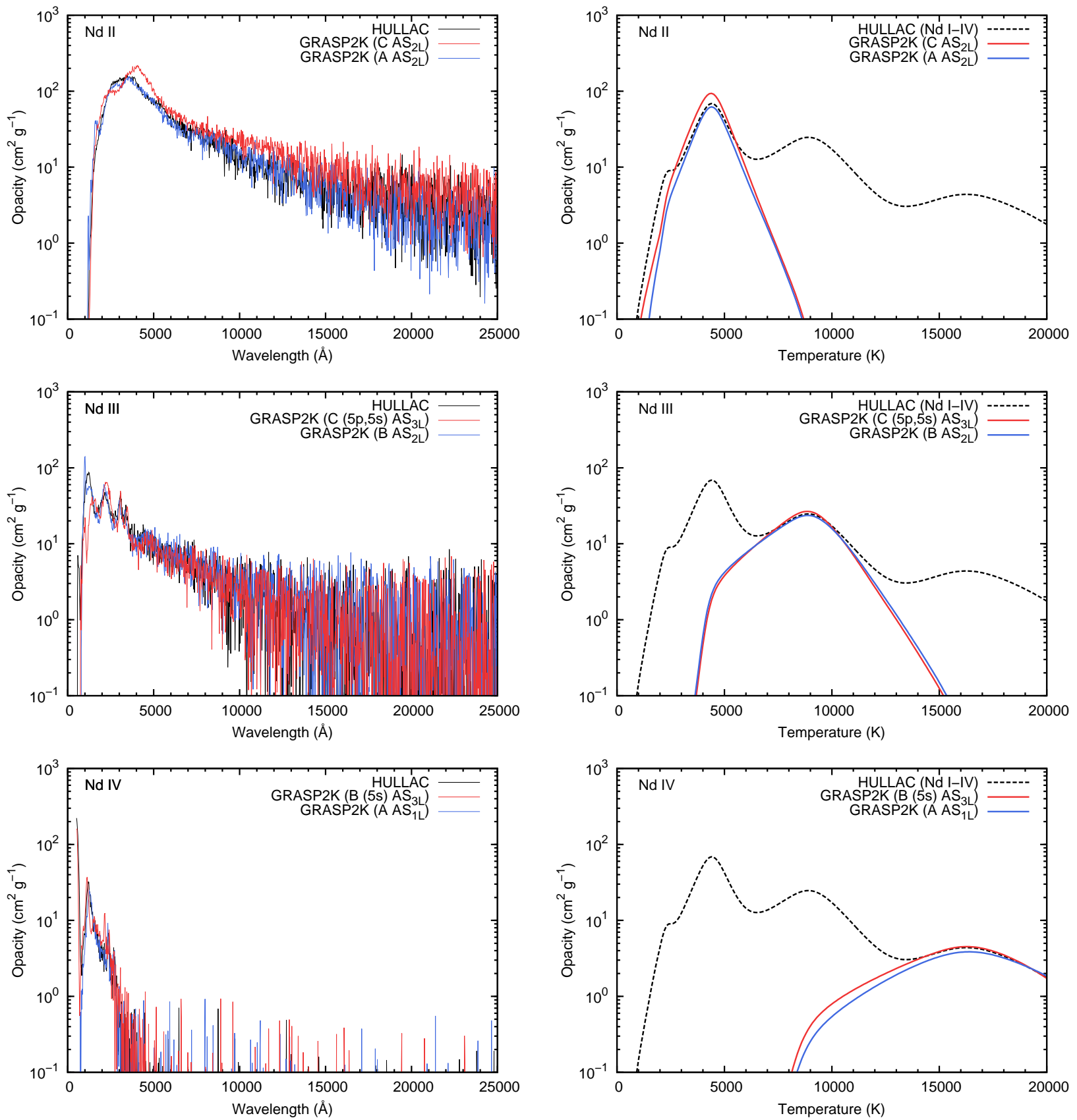

Figure 7. Opacities for Nd II (top), Nd III (middle), and Nd IV (bottom) ions. The left panels show the expansion opacities calculated with $T=5000 \mathrm{~K}, 10000 \mathrm{~K}$, and $15000 \mathrm{~K}$ for Nd II, Nd III, and Nd IV, respectively. The density and time are assumed to be $\rho=1 \times 10^{-13} \mathrm{~g} \mathrm{~cm}^{-3}$ and $t=1$ day after the merger, respectively. The right panels show Planck mean opacities for various temperatures. The dashed curve shows the Planck mean opacities calculated with atomic data for Nd I-IV calculated with the HULLAC code (Tanaka et al. 2018).

\section{REFERENCES}

Abbott, B. P., Abbott, R., Abbott, T. D., et al. 2017a,

Physical Review Letters, 119, 161101

-. 2017b, ApJL, 848, L12

Andreoni, I., Ackley, K., Cooke, J., et al. 2017, PASA, 34, e069
Arcavi, I., Hosseinzadeh, G., Howell, D. A., et al. 2017, Nature, 551, 64

Barnes, J., \& Kasen, D. 2013, ApJ, 775, 18

Chornock, R., Berger, E., Kasen, D., et al. 2017, ApJL, 848, L19 
Table 9. Energy levels $\left(\right.$ in $\mathrm{cm}^{-1}$ ) relative to the ground state for the lowest states of Nd II.

\begin{tabular}{|c|c|c|c|c|}
\hline No. & label & $J$ & $\mathrm{P}$ & $E$ \\
\hline 1 & $4 f^{4}\left({ }_{1}^{5} I\right) 6 s{ }^{6} I$ & $7 / 2$ & + & 0.00 \\
\hline 2 & $4 f^{4}\left({ }_{1}^{5} I\right) 6 s{ }^{6} I$ & $9 / 2$ & + & 547.86 \\
\hline 3 & $4 f^{4}\left({ }_{1}^{5} I\right) 6 s{ }^{6} I$ & $11 / 2$ & + & 1404.36 \\
\hline 4 & $4 f^{4}\left({ }_{1}^{5} I\right) 6 s{ }^{4} I$ & $9 / 2$ & + & 1789.52 \\
\hline 5 & $4 f^{4}\left({ }_{1}^{5} I\right) 6 s{ }^{6} I$ & $13 / 2$ & + & 2425.58 \\
\hline 6 & $4 f^{4}\left({ }_{1}^{5} I\right) 6 s{ }^{4} I$ & $11 / 2$ & + & 3120.96 \\
\hline 7 & $4 f^{4}\left({ }_{1}^{5} I\right) 6 s{ }^{6} I$ & $15 / 2$ & + & 3564.21 \\
\hline 8 & $4 f^{4}\left({ }_{1}^{5} I\right) 5 d^{6} L$ & $11 / 2$ & + & 4019.24 \\
\hline 9 & $4 f^{4}\left({ }_{1}^{5} I\right) 6 s{ }^{4} I$ & $13 / 2$ & + & 4506.07 \\
\hline 10 & $4 f^{4}\left({ }_{1}^{5} I\right) 6 s{ }^{6} I$ & $17 / 2$ & + & 4789.43 \\
\hline 11 & $4 f^{4}\left({ }_{1}^{5} I\right) 5 d^{6} L$ & $13 / 2$ & + & 4941.03 \\
\hline 12 & $4 f^{3}\left({ }_{1}^{4} I\right) 5 d^{2}\left({ }_{2}^{3} F\right){ }^{6} M$ & $13 / 2$ & - & 5477.69 \\
\hline 13 & $4 f^{4}\left({ }_{1}^{5} I\right) 6 s{ }^{4} I$ & $15 / 2$ & + & 5940.53 \\
\hline 14 & $4 f^{4}\left({ }_{1}^{5} I\right) 5 d^{6} L$ & $15 / 2$ & + & 5965.42 \\
\hline 15 & $4 f^{4}\left({ }_{1}^{5} I\right) 5 d^{6} K$ & $9 / 2$ & + & 6065.11 \\
\hline 16 & $4 f^{3}\left({ }_{1}^{4} I\right) 5 d^{2}\left({ }_{2}^{3} F\right){ }^{6} M$ & $15 / 2$ & - & 6750.71 \\
\hline 17 & $4 f^{4}\left({ }_{1}^{5} I\right) 5 d^{6} K$ & $11 / 2$ & + & 6881.31 \\
\hline 18 & $4 f^{4}\left({ }_{1}^{5} I\right) 5 d^{6} L$ & $17 / 2$ & + & 7078.84 \\
\hline 19 & $4 f^{4}\left({ }_{1}^{5} I\right) 5 d^{6} K$ & $13 / 2$ & + & 7802.57 \\
\hline 20 & $4 f^{3}\left({ }_{1}^{4} I\right) 5 d^{2}\left({ }_{2}^{3} F\right)^{6} M$ & $17 / 2$ & - & 8147.61 \\
\hline
\end{tabular}

Note- Table 9 is published in its entirety in the machine-readable format. Part of the values are shown here for guidance regarding its form and content.

Coulter, D. A., Foley, R. J., Kilpatrick, C. D., et al. 2017, Science, 358, 1556

Cowan, R. 1981, The Theory of Atomic Structure and Spectra (University of California Press, Berkeley, CA)

Cowperthwaite, P. S., Berger, E., Villar, V. A., et al. 2017, ApJL, 848, L17

Díaz, M. C., Macri, L. M., Garcia Lambas, D., et al. 2017, ApJL, 848, L29

Drout, M. R., Piro, A. L., Shappee, B. J., et al. 2017, Science, 358, 1570

Dyall, K., Grant, I., Johnson, C., Parpia, F., \& Plummer, E. 1989, Computer Physics Communications, 55, 425

Dzuba, V. A., Safronova, U. I., \& Johnson, W. R. 2003, Phys. Rev. A, 68, 032503

Eastman, R. G., \& Pinto, P. A. 1993, ApJ, 412, 731

Evans, P. A., Cenko, S. B., Kennea, J. A., et al. 2017, Science, 358, 1565
Fernández, R., \& Metzger, B. D. 2016, Annual Review of Nuclear and Particle Science, 66, 23

Fischer, C. F., Godefroid, M., Brage, T., Jönsson, P., \& Gaigalas, G. 2016, Journal of Physics B: Atomic, Molecular and Optical Physics, 49, 182004

Fontes, C. J., Fryer, C. L., Hungerford, A. L., et al. 2017, arXiv:1702.02990, arXiv:1702.02990

Fritzsche, S., \& Grant, I. 1994, Physics Letters A, 186, 152

Gaigalas, G., Fischer, C., Rynkun, P., \& Jönsson, P. 2017, Atoms, 5, 6

Gaigalas, G., \& Rudzikas, Z. 1996, Journal of Physics B: Atomic, Molecular and Optical Physics, 29, 3303

Gaigalas, G., Rudzikas, Z., \& Fischer, C. F. 1997, Journal of Physics B: Atomic, Molecular and Optical Physics, 30, 3747

Grant, I. P. 1974, Journal of Physics B: Atomic and Molecular Physics, 7, 1458 
Table 10. Transition energies $\Delta E$ (in $\mathrm{cm}^{-1}$ ), transition wavelengths $\lambda$ (in $\AA$ ), line strengths $S$ (in a.u.), weighted oscillator strengths $g f$ and transition rates $A$ (in s ${ }^{-1}$ ) for $\mathrm{E} 1$ transitions of $\mathrm{Nd}$ II ion. All transition data are in length form. $d T$ is the relative difference of the transition rates in length and velocity form as given by Eq. (5).

\begin{tabular}{|c|c|c|c|c|c|c|c|}
\hline upper & lower & $\Delta E$ & $\lambda$ & $S$ & $g f$ & $A$ & $d T$ \\
\hline $4 f^{3}\left({ }_{1}^{4} I\right) 5 d^{2}\left({ }_{2}^{3} F\right){ }^{6} L_{11 / 2}$ & $4 f^{3}\left({ }_{1}^{4} I\right) 5 d{ }^{5} K 6 p{ }^{6} L_{11 / 2}$ & 22745 & 4396.48 & $4.593 \mathrm{E}+00$ & $3.173 \mathrm{E}-01$ & $9.127 \mathrm{E}+06$ & 0.050 \\
\hline $4 f^{3}\left({ }_{1}^{4} I\right) 5 d^{2}\left({ }_{2}^{3} F\right){ }^{6} L_{11 / 2}$ & $4 f^{3}\left({ }_{1}^{4} I\right) 5 d^{5} L 6 p^{6} L_{11 / 2}$ & 23391 & 4275.02 & $1.001 \mathrm{E}+01$ & $7.115 \mathrm{E}-01$ & $2.164 \mathrm{E}+07$ & 0.154 \\
\hline $4 f^{3}\left({ }_{1}^{4} I\right) 5 d^{2}\left({ }_{2}^{3} F\right){ }^{6} L_{11 / 2}$ & $4 f^{3}\left({ }_{1}^{4} I\right) 5 d^{5} K 6 p^{6} K_{11 / 2}$ & 24959 & 4006.51 & $7.628 \mathrm{E}-01$ & $5.783 \mathrm{E}-02$ & $2.002 \mathrm{E}+06$ & 0.130 \\
\hline $4 f^{3}\left({ }_{1}^{4} I\right) 5 d^{2}\left({ }_{2}^{3} F\right){ }^{6} L_{11 / 2}$ & $4 f^{3}\left({ }_{1}^{4} I\right) 5 d^{5} K 6 p{ }^{6} L_{11 / 2}$ & 25373 & 3941.14 & $1.380 \mathrm{E}+00$ & $1.063 \mathrm{E}-01$ & $3.807 \mathrm{E}+06$ & 0.033 \\
\hline $4 f^{3}\left({ }_{1}^{4} I\right) 5 d^{2}\left({ }_{2}^{3} F\right){ }^{6} L_{11 / 2}$ & $4 f^{3}\left({ }_{1}^{4} I\right) 5 d^{5} K 6 p{ }^{6} I_{11 / 2}$ & 26882 & 3719.95 & $2.049 \mathrm{E}-01$ & $1.673 \mathrm{E}-02$ & $6.722 \mathrm{E}+05$ & 0.041 \\
\hline $4 f^{3}\left({ }_{1}^{4} I\right) 5 d^{2}\left({ }_{2}^{3} F\right){ }^{6} L_{11 / 2}$ & $4 f^{3}\left({ }_{1}^{4} I\right) 5 d{ }^{5} L 6 p{ }^{6} K_{11 / 2}$ & 27650 & 3616.62 & $1.213 \mathrm{E}-01$ & $1.018 \mathrm{E}-02$ & $4.330 \mathrm{E}+05$ & 0.069 \\
\hline $4 f^{3}\left({ }_{1}^{4} I\right) 5 d^{2}\left({ }_{2}^{3} F\right){ }^{6} L_{11 / 2}$ & $4 f^{3}\left({ }_{1}^{4} I\right) 5 d^{5} K 6 p^{6} I_{11 / 2}$ & 28842 & 3467.14 & $5.807 \mathrm{E}-02$ & $5.088 \mathrm{E}-03$ & $2.352 \mathrm{E}+05$ & 0.351 \\
\hline $4 f^{3}\left({ }_{1}^{4} I\right) 5 d^{2}\left({ }_{2}^{3} F\right){ }^{6} L_{11 / 2}$ & $4 f^{3}\left({ }_{1}^{4} I\right) 5 d^{5} I 6 p{ }^{6} K_{11 / 2}$ & 29386 & 3402.93 & $3.867 \mathrm{E}-01$ & $3.452 \mathrm{E}-02$ & $1.657 \mathrm{E}+06$ & 0.120 \\
\hline $4 f^{3}\left({ }_{1}^{4} I\right) 5 d^{2}\left({ }_{2}^{3} F\right){ }^{6} L_{11 / 2}$ & $4 f^{3}\left({ }_{1}^{4} I\right) 5 d^{3} I 6 p{ }^{4} K_{11 / 2}$ & 29450 & 3395.57 & $1.159 \mathrm{E}-02$ & $1.037 \mathrm{E}-03$ & $5.002 \mathrm{E}+04$ & 0.124 \\
\hline $4 f^{3}\left({ }_{1}^{4} I\right) 5 d^{2}\left({ }_{2}^{3} F\right){ }^{6} L_{11 / 2}$ & $4 f^{3}\left({ }_{1}^{4} I\right) 5 d^{5} I 6 p{ }^{6} K_{11 / 2}$ & 30233 & 3307.58 & $5.567 \mathrm{E}-02$ & $5.112 \mathrm{E}-03$ & $2.597 \mathrm{E}+05$ & 0.164 \\
\hline $4 f^{3}\left({ }_{1}^{4} I\right) 5 d^{2}\left({ }_{2}^{3} F\right){ }^{6} L_{11 / 2}$ & $4 f^{3}\left({ }_{1}^{4} I\right) 5 d^{3} I 6 p{ }^{4} H_{11 / 2}$ & 30410 & 3288.31 & $3.589 \mathrm{E}-02$ & $3.316 \mathrm{E}-03$ & $1.704 \mathrm{E}+05$ & 0.320 \\
\hline $4 f^{3}\left({ }_{1}^{4} I\right) 5 d^{2}\left({ }_{2}^{3} F\right){ }^{6} L_{11 / 2}$ & $4 f^{3}\left({ }_{1}^{4} I\right) 5 d^{5} I 6 p{ }^{6} I_{11 / 2}$ & 30982 & 3227.58 & $2.441 \mathrm{E}-04$ & $2.297 \mathrm{E}-05$ & $1.226 \mathrm{E}+03$ & 0.999 \\
\hline $4 f^{3}\left({ }_{1}^{4} I\right) 5 d^{2}\left({ }_{2}^{3} F\right){ }^{6} L_{11 / 2}$ & $4 f^{3}\left({ }_{1}^{4} I\right) 5 d^{5} I 6 p{ }^{6} I_{11 / 2}$ & 31155 & 3209.69 & $5.477 \mathrm{E}-02$ & $5.184 \mathrm{E}-03$ & $2.797 \mathrm{E}+05$ & 0.186 \\
\hline $4 f^{3}\left({ }_{1}^{4} I\right) 5 d^{2}\left({ }_{2}^{3} F\right){ }^{6} L_{11 / 2}$ & $4 f^{3}\left({ }_{1}^{4} I\right) 5 d^{5} I 6 p{ }^{4} K_{11 / 2}$ & 31541 & 3170.46 & $3.420 \mathrm{E}-02$ & $3.276 \mathrm{E}-03$ & $1.812 \mathrm{E}+05$ & 0.373 \\
\hline $4 f^{3}\left({ }_{1}^{4} I\right) 5 d^{2}\left({ }_{2}^{3} F\right){ }^{6} L_{11 / 2}$ & $4 f^{3}\left({ }_{1}^{4} I\right) 5 d^{5} I 6 p{ }^{4} H_{11 / 2}$ & 31848 & 3139.89 & $2.205 \mathrm{E}-04$ & $2.133 \mathrm{E}-05$ & $1.202 \mathrm{E}+03$ & 0.541 \\
\hline $4 f^{3}\left({ }_{1}^{4} I\right) 5 d^{2}\left({ }_{2}^{3} F\right){ }^{6} L_{11 / 2}$ & $4 f^{3}\left({ }_{1}^{4} I\right) 5 d^{3} H 6 p^{4} I_{11 / 2}$ & 32432 & 3083.34 & $5.245 \mathrm{E}-03$ & $5.167 \mathrm{E}-04$ & $3.021 \mathrm{E}+04$ & 0.319 \\
\hline $4 f^{3}\left({ }_{1}^{4} I\right) 5 d^{2}\left({ }_{2}^{3} F\right){ }^{6} L_{11 / 2}$ & $4 f^{3}\left({ }_{1}^{4} I\right) 5 d^{5} I 6 p{ }^{6} H_{11 / 2}$ & 33244 & 3008.02 & $2.221 \mathrm{E}-03$ & $2.242 \mathrm{E}-04$ & $1.377 \mathrm{E}+04$ & 0.427 \\
\hline $4 f^{3}\left({ }_{1}^{4} I\right) 5 d^{2}\left({ }_{2}^{3} F\right){ }^{6} L_{11 / 2}$ & $4 f^{3}\left({ }_{1}^{4} I\right) 5 d{ }^{5} H 6 p{ }^{6} H_{11 / 2}$ & 33332 & 3000.04 & $1.985 \mathrm{E}-05$ & $2.010 \mathrm{E}-06$ & $1.241 \mathrm{E}+02$ & 0.938 \\
\hline $4 f^{3}\left({ }_{1}^{4} I\right) 5 d^{2}\left({ }_{2}^{3} F\right){ }^{6} L_{11 / 2}$ & $4 f^{3}\left({ }_{1}^{4} I\right) 5 d{ }^{5} H 6 p{ }^{6} G_{11 / 2}$ & 33384 & 2995.44 & $1.588 \mathrm{E}-04$ & $1.611 \mathrm{E}-05$ & $9.980 \mathrm{E}+02$ & 0.446 \\
\hline $4 f^{3}\left({ }_{1}^{4} I\right) 5 d^{2}\left({ }_{2}^{3} F\right){ }^{6} L_{11 / 2}$ & $4 f^{3}\left({ }_{1}^{4} I\right) 5 d^{5} I 6 p^{6} I_{11 / 2}$ & 33499 & 2985.08 & $8.480 \mathrm{E}-04$ & $8.629 \mathrm{E}-05$ & $5.383 \mathrm{E}+03$ & 0.424 \\
\hline
\end{tabular}

Note - Table 10 is published in its entirety in the machine-readable format. Part of the values are shown here for guidance regarding its form and content.

—. 2007, Relativistic Quantum Theory of Atoms and Molecules (Springer, New York)

Hotokezaka, K., Beniamini, P., \& Piran, T. 2018, ArXiv e-prints, arXiv:1801.01141

Jönsson, P., Gaigalas, G., Bieroń, J., Fischer, C. F., \& Grant, I. 2013, Computer Physics Communications, 184, 2197

Karp, A. H., Lasher, G., Chan, K. L., \& Salpeter, E. E. 1977, ApJ, 214, 161

Kasen, D., Badnell, N. R., \& Barnes, J. 2013, ApJ, 774, 25

Kasen, D., Fernández, R., \& Metzger, B. D. 2015, MNRAS, 450, 1777

Kasen, D., Metzger, B., Barnes, J., Quataert, E., \& Ramirez-Ruiz, E. 2017, Nature, 551, 80

Kasen, D., Thomas, R. C., \& Nugent, P. 2006, ApJ, 651, 366

Kasliwal, M. M., Nakar, E., Singer, L. P., et al. 2017, Science, 358, 1559
Kilpatrick, C. D., Foley, R. J., Kasen, D., et al. 2017, Science, 358, 1583

Kulkarni, S. R. 2005, arXiv:astro-ph/0510256, arXiv:astro-ph/0510256

Li, L.-X., \& Paczyński, B. 1998, ApJL, 507, L59

Lipunov, V. M., Gorbovskoy, E., Kornilov, V. G., et al. 2017, ApJL, 850, L1

McCully, C., Hiramatsu, D., Howell, D. A., et al. 2017, ApJL, 848, L32

McKenzie, B., Grant, I., \& Norrington, P. 1980, Computer Physics Communications, 21, 233

Metzger, B. D. 2017, Living Reviews in Relativity, 20, 3

Metzger, B. D., \& Fernández, R. 2014, MNRAS, 441, 3444

Metzger, B. D., Martínez-Pinedo, G., Darbha, S., et al. 2010, MNRAS, 406, 2650

Nicholl, M., Berger, E., Kasen, D., et al. 2017, ApJL, 848, L18 
Table 11. Energy levels $\left(\right.$ in $\mathrm{cm}^{-1}$ ) relative to the ground state for the lowest states of Nd III.

\begin{tabular}{rlrrr}
\hline \hline No. & \multicolumn{1}{c}{ label } & $J$ & $\mathrm{P}$ & \multicolumn{1}{c}{$E$} \\
\hline 1 & $4 f^{4}\left({ }_{1}^{5} I\right){ }^{5} I$ & 4 & + & 0.00 \\
2 & $4 f^{4}\left({ }_{1}^{5} I\right){ }^{5} I$ & 5 & + & 1072.58 \\
3 & $4 f^{4}\left({ }_{1}^{5} I\right){ }^{5} I$ & 6 & + & 2263.93 \\
4 & $4 f^{4}\left({ }_{1}^{5} I\right){ }^{5} I$ & 7 & + & 3542.92 \\
5 & $4 f^{4}\left({ }_{1}^{5} I\right){ }^{5} I$ & 8 & + & 4884.66 \\
6 & $4 f^{4}\left({ }_{1}^{5} F\right){ }^{5} F$ & 1 & + & 11739.04 \\
7 & $4 f^{4}\left({ }_{1}^{5} F\right){ }^{5} F$ & 2 & + & 12098.57 \\
8 & $4 f^{4}\left({ }_{1}^{5} F\right){ }^{5} F$ & 3 & + & 12724.33 \\
9 & $4 f^{4}\left({ }_{0}^{5} S\right){ }^{5} S$ & 2 & + & 13433.63 \\
10 & $4 f^{4}\left({ }_{1}^{5} F\right){ }^{5} F$ & 4 & + & 13459.96 \\
11 & $4 f^{4}\left({ }_{1}^{5} F\right){ }^{5} F$ & 5 & + & 14444.26 \\
12 & $4 f^{4}\left({ }_{2}^{3} K\right){ }^{3} K$ & 6 & + & 15065.97 \\
13 & $4 f^{3}\left({ }_{1}^{4} I\right) 5 d d^{5} K$ & 5 & - & 15128.43 \\
14 & $4 f^{3}\left({ }_{1}^{4} I\right) 5 d{ }^{5} L$ & 6 & - & 15257.69 \\
15 & $4 f^{4}\left({ }_{4}^{3} H\right){ }^{3} H$ & 4 & + & 16151.40 \\
16 & $4 f^{4}\left({ }_{2}^{3} K\right){ }^{3} K$ & 7 & + & 16180.31 \\
17 & $4 f^{3}\left({ }_{1}^{4} I\right) 5 d{ }^{5} K$ & 6 & - & 16720.95 \\
18 & $4 f^{3}\left({ }_{1}^{4} I\right) 5 d{ }^{5} L$ & 7 & - & 16985.08 \\
19 & $4 f^{4}\left({ }_{1}^{5} G\right){ }^{5} G$ & 2 & + & 17295.05 \\
20 & $4 f^{4}\left({ }_{1}^{5} G\right){ }^{5} G$ & 3 & + & 17368.09 \\
\hline & & & \\
\hline
\end{tabular}

Note- Table 11 is published in its entirety in the machine-readable format. Part of the values are shown here for guidance regarding its form and content.

Olsen, J., Godefroid, M. R., Jönsson, P., Malmqvist, P. A., \& Fischer, C. F. 1995, Phys. Rev. E, 52, 4499

Perego, A., Radice, D., \& Bernuzzi, S. 2017, ApJL, 850, L37

Pian, E., D'Avanzo, P., Benetti, S., et al. 2017, Nature, 551, 67

Radziemski, L. J., Fisher, K., \& Steinhaus, D. 1970

Rosswog, S. 2015, International Journal of Modern Physics D, 24, 1530012

Rosswog, S., Sollerman, J., Feindt, U., et al. 2017, arXiv:1710.05445, arXiv:1710.05445

Shappee, B. J., Simon, J. D., Drout, M. R., et al. 2017, Science, 358, 1574

Shibata, M., Fujibayashi, S., Hotokezaka, K., et al. 2017, PhRvD, 96, 123012

Siebert, M. R., Foley, R. J., Drout, M. R., et al. 2017, ApJL, 848, L26
Smartt, S. J., Chen, T.-W., Jerkstrand, A., et al. 2017, Nature, 551, 75

Soares-Santos, M., Holz, D. E., Annis, J., et al. 2017, ApJL, 848, L16

Tanaka, M. 2016, Advances in Astronomy, 2016, 634197

Tanaka, M., \& Hotokezaka, K. 2013, ApJ, 775, 113

Tanaka, M., Hotokezaka, K., Kyutoku, K., et al. 2014, ApJ, 780,31

Tanaka, M., Utsumi, Y., Mazzali, P. A., et al. 2017, PASJ, 69, 102

Tanaka, M., Kato, D., Gaigalas, G., et al. 2018, ApJ, 852, 109

Tanvir, N. R., Levan, A. J., González-Fernández, C., et al. 2017, ApJL, 848, L27

Tominaga, N., Tanaka, M., Morokuma, T., et al. 2018, PASJ, arXiv:1710.05865 
Table 12. Transition energies $\Delta E$ (in $\mathrm{cm}^{-1}$ ), transition wavelengths $\lambda$ (in $\AA$ ), line strengths $S$ (in a.u.), weighted oscillator strengths $g f$ and transition rates $A$ (in s ${ }^{-1}$ ) for E1 transitions of Nd III ion. All transition data are in length form. $d T$ is the relative difference of the transition rates in length and velocity form as given by Eq. (5).

\begin{tabular}{|c|c|c|c|c|c|c|c|}
\hline upper & lower & $\Delta E$ & $\lambda$ & $S$ & $g f$ & $A$ & $d T$ \\
\hline $4 f^{4}\left({ }_{2}^{3} P\right)^{3} P_{0}$ & $4 f^{3}\left({ }_{1}^{4} F\right) 5 d^{5} D_{1}$ & 6521 & 15334.41 & $2.338 \mathrm{E}-03$ & $4.632 \mathrm{E}-05$ & $4.380 \mathrm{E}+02$ & 0.948 \\
\hline $4 f^{4}\left({ }_{2}^{3} P\right){ }^{3} P_{0}$ & $4 f^{3}\left({ }_{1}^{4} F\right) 5 d^{3} P_{1}$ & 9156 & 10920.99 & $1.996 \mathrm{E}-02$ & $5.553 \mathrm{E}-04$ & $1.035 \mathrm{E}+04$ & 0.861 \\
\hline $4 f^{4}\left({ }_{2}^{3} P\right)^{3} P_{0}$ & $4 f^{3}\left({ }_{1}^{4} F\right) 5 d^{5} P_{1}$ & 10206 & 9798.04 & $5.262 \mathrm{E}-03$ & $1.631 \mathrm{E}-04$ & $3.778 \mathrm{E}+03$ & 0.787 \\
\hline $4 f^{4}\left({ }_{2}^{3} P\right)^{3} P_{0}$ & $4 f^{3}\left({ }_{1}^{4} F\right) 5 d^{5} F_{1}$ & 10697 & 9348.19 & $8.930 \mathrm{E}-03$ & $2.901 \mathrm{E}-04$ & $7.382 \mathrm{E}+03$ & 0.822 \\
\hline $4 f^{4}\left({ }_{2}^{3} P\right){ }^{3} P_{0}$ & $4 f^{3}\left({ }_{1}^{4} S\right) 5 d^{5} D_{1}$ & 11516 & 8683.29 & $3.115 \mathrm{E}-05$ & $1.090 \mathrm{E}-06$ & $3.214 \mathrm{E}+01$ & 0.890 \\
\hline $4 f^{4}\left({ }_{2}^{3} P\right)^{3} P_{0}$ & $4 f^{3}\left({ }_{1}^{4} S\right) 5 d^{3} D_{1}$ & 12113 & 8255.57 & $1.272 \mathrm{E}-02$ & $4.681 \mathrm{E}-04$ & $1.527 \mathrm{E}+04$ & 0.752 \\
\hline $4 f^{4}\left({ }_{2}^{3} P\right)^{3} P_{0}$ & $4 f^{3}\left({ }_{1}^{4} F\right) 5 d^{3} D_{1}$ & 14250 & 7017.52 & $9.589 \mathrm{E}-02$ & $4.150 \mathrm{E}-03$ & $1.874 \mathrm{E}+05$ & 0.710 \\
\hline $4 f^{4}\left({ }_{2}^{3} P\right)^{3} P_{0}$ & $4 f^{3}\left({ }_{1}^{2} G\right) 5 d^{3} D_{1}$ & 17425 & 5738.84 & $7.686 \mathrm{E}-02$ & $4.068 \mathrm{E}-03$ & $2.746 \mathrm{E}+05$ & 0.647 \\
\hline $4 f^{4}\left({ }_{2}^{3} P\right){ }^{3} P_{0}$ & $4 f^{3}\left({ }_{1}^{4} G\right) 5 d^{5} F_{1}$ & 18400 & 5434.62 & $5.429 \mathrm{E}-04$ & $3.034 \mathrm{E}-05$ & $2.284 \mathrm{E}+03$ & 0.634 \\
\hline $4 f^{4}\left({ }_{2}^{3} P\right)^{3} P_{0}$ & $4 f^{3}\left({ }_{1}^{2} P\right) 5 d^{3} P_{1}$ & 19753 & 5062.36 & $1.351 \mathrm{E}-02$ & $8.110 \mathrm{E}-04$ & $7.036 \mathrm{E}+04$ & 0.786 \\
\hline $4 f^{4}\left({ }_{2}^{3} P\right)^{3} P_{0}$ & $4 f^{3}\left({ }_{1}^{4} G\right) 5 d^{5} D_{1}$ & 20499 & 4878.06 & $4.216 \mathrm{E}-03$ & $2.625 \mathrm{E}-04$ & $2.453 \mathrm{E}+04$ & 0.562 \\
\hline $4 f^{4}\left({ }_{2}^{3} P\right)^{3} P_{0}$ & $4 f^{3}\left({ }_{1}^{2} D\right) 5 d^{3} P_{1}$ & 20588 & 4857.14 & $6.608 \mathrm{E}-02$ & $4.132 \mathrm{E}-03$ & $3.894 \mathrm{E}+05$ & 0.679 \\
\hline $4 f^{4}\left({ }_{2}^{3} P\right){ }^{3} P_{0}$ & $4 f^{3}\left({ }_{1}^{2} D\right) 5 d^{3} S_{1}$ & 21850 & 4576.58 & $4.184 \mathrm{E}-02$ & $2.777 \mathrm{E}-03$ & $2.948 \mathrm{E}+05$ & 0.711 \\
\hline $4 f^{4}\left({ }_{2}^{3} P\right)^{3} P_{0}$ & $4 f^{3}\left({ }_{1}^{4} F\right) 6 s{ }^{5} F_{1}$ & 22166 & 4511.37 & $1.708 \mathrm{E}-05$ & $1.150 \mathrm{E}-06$ & $1.257 \mathrm{E}+02$ & 0.812 \\
\hline $4 f^{4}\left({ }_{2}^{3} P\right)^{3} P_{0}$ & $4 f^{3}\left({ }_{1}^{2} D\right) 5 d^{3} S_{1}$ & 22582 & 4428.21 & $7.961 \mathrm{E}-03$ & $5.461 \mathrm{E}-04$ & $6.192 \mathrm{E}+04$ & 0.707 \\
\hline $4 f^{4}\left({ }_{2}^{3} P\right)^{3} P_{0}$ & $4 f^{3}\left({ }_{1}^{2} P\right) 5 d^{3} D_{1}$ & 23964 & 4172.76 & $1.943 \mathrm{E}-02$ & $1.415 \mathrm{E}-03$ & $1.806 \mathrm{E}+05$ & 0.137 \\
\hline $4 f^{4}\left({ }_{2}^{3} P\right)^{3} P_{0}$ & $4 f^{3}\left({ }_{1}^{4} S\right) 6 s^{3} S_{1}$ & 24808 & 4030.93 & $4.263 \mathrm{E}-04$ & $3.213 \mathrm{E}-05$ & $4.396 \mathrm{E}+03$ & 0.318 \\
\hline $4 f^{4}\left({ }_{2}^{3} P\right)^{3} P_{0}$ & $4 f^{3}\left({ }_{1}^{4} S\right) 6 s^{3} S_{1}$ & 25324 & 3948.70 & $3.464 \mathrm{E}-03$ & $2.665 \mathrm{E}-04$ & $3.800 \mathrm{E}+04$ & 0.784 \\
\hline $4 f^{4}\left({ }_{2}^{3} P\right)^{3} P_{0}$ & $4 f^{3}\left({ }_{1}^{2} D\right) 5 d{ }^{1} P_{1}$ & 26223 & 3813.37 & $2.834 \mathrm{E}-03$ & $2.257 \mathrm{E}-04$ & $3.452 \mathrm{E}+04$ & 0.389 \\
\hline $4 f^{4}\left({ }_{2}^{3} P\right)^{3} P_{0}$ & $4 f^{3}\left({ }_{1}^{4} D\right) 5 d^{5} F_{1}$ & 27992 & 3572.45 & $4.279 \mathrm{E}-09$ & $3.638 \mathrm{E}-10$ & $6.339 \mathrm{E}-02$ & 0.999 \\
\hline
\end{tabular}

Note - Table 12 is published in its entirety in the machine-readable format. Part of the values are shown here for guidance regarding its form and content.

Troja, E., Piro, L., van Eerten, H., et al. 2017, Nature, 551, 71

Utsumi, Y., Tanaka, M., Tominaga, N., et al. 2017, PASJ, 69,101

Valenti, S., David, Sand, J., et al. 2017, ApJL, 848, L24

Wollaeger, R. T., Korobkin, O., Fontes, C. J., et al. 2017, arXiv:1705.07084, arXiv:1705.07084

Wyart, J.-F. 2010, Physica Scripta, 82, 035302
Wyart, J.-F., Meftah, A., Bachelier, A., et al. 2006, Journal of Physics B: Atomic, Molecular and Optical Physics, 39, L77

Wyart, J.-F., Meftah, A., Sinzelle, J., et al. 2008, Journal of Physics B: Atomic, Molecular and Optical Physics, 41, 085001

Wyart, J.-F., Meftah, A., Tchang-Brillet, W.-. L., et al. 2007, Journal of Physics B: Atomic, Molecular and Optical Physics, 40, 3957

Yoca, S. E., \& Quinet, P. 2014, Journal of Physics B: Atomic, Molecular and Optical Physics, 47, 035002 Zhang, Z. G., Svanberg, S., Palmeri, P., Quinet, P., \& Biémont, E. 2002, A\&A, 385, 724 

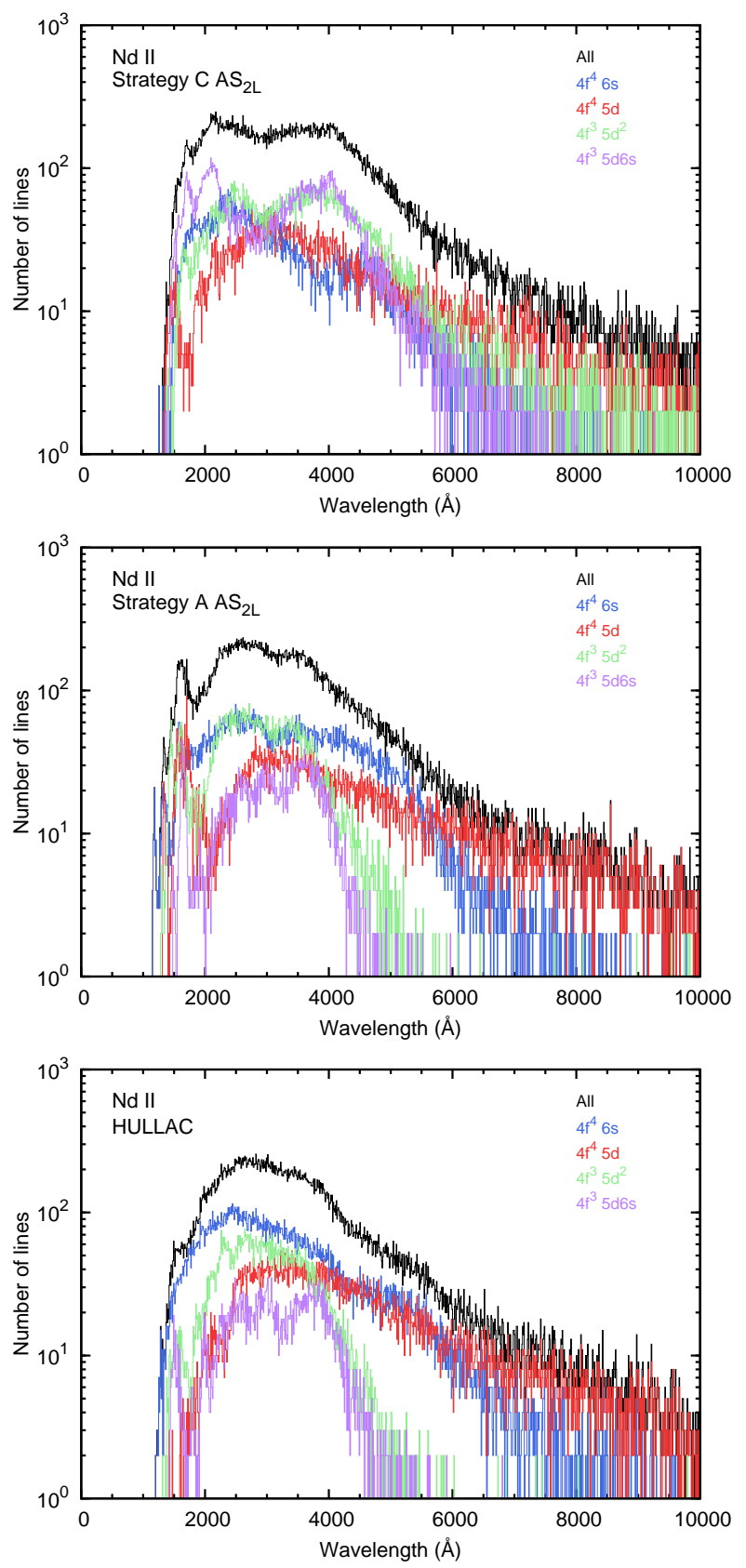

Figure 8. Number of strong transitions of Nd II as a function of wavelengths. The strong transitions are selected by the criterion of $g_{l} f_{l} \exp \left(-E_{l} / k T\right)>10^{-5}$ with $T=5000 \mathrm{~K}$. The black lines show total number of strong transitions while color lines show transitions from each lower-level configuration. 

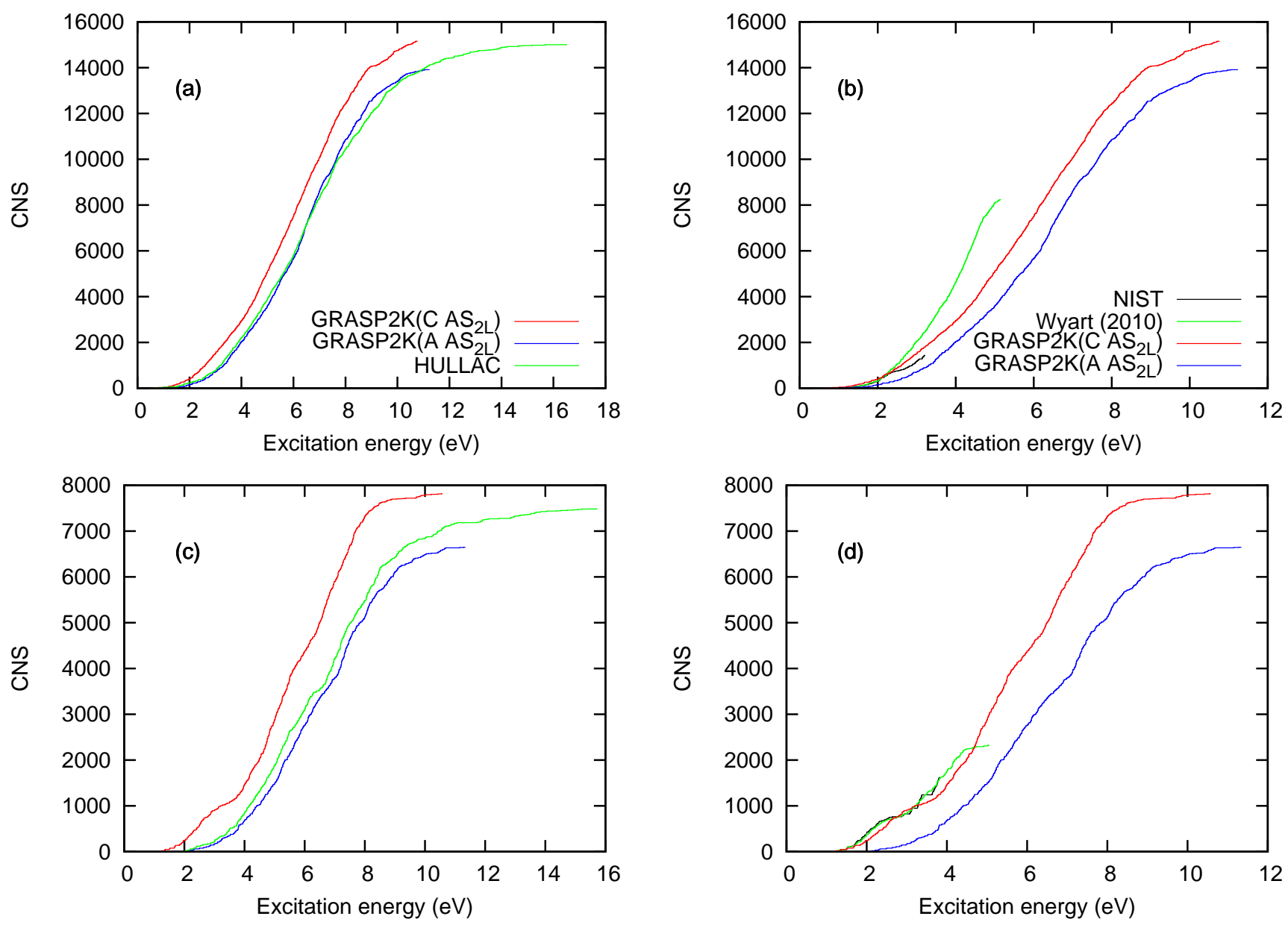

Figure 9. Cumulative number of states for $4 f^{3} 5 d^{2}$ (a,b) and $4 f^{3} 5 d 6 s$ (c,d) configurations as a function of excitation energy. $(\mathrm{a}, \mathrm{c})$ : Results with the present and previous GRASP2K and HULLAC calculations. (b,d): Results with the present GRASP2K calculation, NIST, and the semi-empirical method by Wyart (2010). 
Table 13. Energy levels $\left(\right.$ in $\mathrm{cm}^{-1}$ ) relative to the ground state for the lowest states of $\mathrm{Nd}$ IV.

\begin{tabular}{rcrlr}
\hline \hline No. & label & \multicolumn{1}{c}{$J$} & \multicolumn{1}{c}{ P } \\
\hline 1 & $4 f^{3}\left({ }_{1}^{4} I\right)^{4} I$ & $9 / 2$ & - & 0.00 \\
2 & $4 f^{3}\left({ }_{1}^{4} I\right)^{4} I$ & $11 / 2$ & - & 1748.59 \\
3 & $4 f^{3}\left({ }_{1}^{4} I\right){ }^{4} I$ & $13 / 2$ & - & 3627.05 \\
4 & $4 f^{3}\left({ }_{1}^{4} I\right)^{4} I$ & $15 / 2$ & - & 5595.78 \\
5 & $4 f^{3}\left({ }_{1}^{4} F\right)^{4} F$ & $3 / 2$ & - & 13076.19 \\
6 & $4 f^{3}\left({ }_{2}^{2} H\right)^{2} H$ & $9 / 2$ & - & 13536.44 \\
7 & $4 f^{3}\left({ }_{1}^{4} F\right)^{4} F$ & $5 / 2$ & - & 14022.10 \\
8 & $4 f^{3}\left({ }_{1}^{4} S\right)^{4} S$ & $3 / 2$ & - & 14617.16 \\
9 & $4 f^{3}\left({ }_{1}^{4} F\right)^{4} F$ & $7 / 2$ & - & 14911.07 \\
10 & $4 f^{3}\left({ }_{1}^{4} F\right)^{4} F$ & $9 / 2$ & - & 15979.23 \\
11 & $4 f^{3}\left({ }_{2}^{2} H\right)^{2} H$ & $11 / 2$ & - & 16581.13 \\
12 & $4 f^{3}\left({ }_{1}^{2} G\right){ }^{2} G$ & $7 / 2$ & - & 18780.28 \\
13 & $4 f^{3}\left({ }_{1}^{4} G\right){ }^{4} G$ & $5 / 2$ & - & 19780.48 \\
14 & $4 f^{3}\left({ }_{1}^{4} G\right){ }^{4} G$ & $9 / 2$ & - & 21211.00 \\
15 & $4 f^{3}\left({ }_{1}^{4} G\right){ }^{4} G$ & $7 / 2$ & - & 21217.90 \\
16 & $4 f^{3}\left({ }_{1}^{2} K\right)^{2} K$ & $13 / 2$ & - & 21444.78 \\
17 & $4 f^{3}\left({ }_{1}^{4} G\right){ }^{4} G$ & $9 / 2$ & - & 22708.88 \\
18 & $4 f^{3}\left({ }_{1}^{2} D\right){ }^{2} D$ & $3 / 2$ & - & 23160.03 \\
19 & $4 f^{3}\left({ }_{1}^{2} K\right)^{2} K$ & $15 / 2$ & - & 23397.52 \\
20 & $4 f^{3}\left({ }_{1}^{4} G\right){ }^{4} G$ & $11 / 2$ & - & 23768.32 \\
\hline
\end{tabular}

Note- Table 13 is published in its entirety in the machine-readable format. Part of the values are shown here for guidance regarding its form and content. 
Table 14. Transition energies $\Delta E$ (in $\mathrm{cm}^{-1}$ ), transition wavelengths $\lambda$ (in $\AA$ ), line strengths $S$ (in a.u.), weighted oscillator strengths $g f$ and transition rates $A$ (in s ${ }^{-1}$ ) for E1 transitions of $\mathrm{Nd}$ IV ion. All transition data are in length form. $d T$ is the relative difference of the transition rates in length and velocity form as given by Eq. (5).

\begin{tabular}{|c|c|c|c|c|c|c|c|}
\hline upper & lower & $\Delta E$ & $\lambda$ & $S$ & $g f$ & $A$ & $d T$ \\
\hline $4 f^{3}\left({ }_{1}^{2} P\right) 5 p^{6}{ }^{2} P_{1 / 2}$ & $4 f^{2}\left({ }_{1}^{3} F\right) 5 p^{6} 5 d^{2} P_{1 / 2}$ & 52003 & 1922.95 & $3.607 \mathrm{E}-02$ & $5.698 \mathrm{E}-03$ & $5.140 \mathrm{E}+06$ & 0.422 \\
\hline $4 f^{3}\left({ }_{1}^{4} D\right) 5 p^{6}{ }^{4} D_{1 / 2}$ & $4 f^{2}\left({ }_{1}^{3} F\right) 5 p^{6} 5 d^{2} P_{1 / 2}$ & 44283 & 2258.20 & $7.141 \mathrm{E}-03$ & $9.606 \mathrm{E}-04$ & $6.283 \mathrm{E}+05$ & 0.235 \\
\hline $4 f^{2}\left({ }_{1}^{3} F\right) 5 p^{6} 5 d^{2} P_{1 / 2}$ & $4 f^{4}\left({ }_{1}^{5} F\right) 5 p^{5} \quad{ }^{6} D_{1 / 2}$ & 83619 & 1195.89 & $3.829 \mathrm{E}-07$ & $9.725 \mathrm{E}-08$ & $2.268 \mathrm{E}+02$ & 0.945 \\
\hline $4 f^{2}\left({ }_{1}^{3} F\right) 5 p^{6} 5 d^{2} P_{1 / 2}$ & $4 f^{4}\left({ }_{1}^{5} G\right) 5 p^{5} \quad{ }^{6} F_{1 / 2}$ & 85201 & 1173.69 & $2.099 \mathrm{E}-05$ & $5.432 \mathrm{E}-06$ & $1.315 \mathrm{E}+04$ & 0.116 \\
\hline $4 f^{2}\left({ }_{1}^{3} F\right) 5 p^{6} 5 d^{2} P_{1 / 2}$ & $4 f^{2}\left({ }_{1}^{3} F\right) 5 p^{6} 6 p^{4} D_{1 / 2}$ & 92307 & 1083.33 & $1.546 \mathrm{E}-05$ & $4.336 \mathrm{E}-06$ & $1.232 \mathrm{E}+04$ & 0.249 \\
\hline $4 f^{2}\left({ }_{1}^{3} F\right) 5 p^{6} 5 d^{2} P_{1 / 2}$ & $4 f^{4}\left({ }_{1}^{3} D\right) 5 p^{5}{ }^{4} P_{1 / 2}$ & 96617 & 1035.01 & $2.052 \mathrm{E}-05$ & $6.022 \mathrm{E}-06$ & $1.875 \mathrm{E}+04$ & 0.739 \\
\hline $4 f^{2}\left({ }_{1}^{3} F\right) 5 p^{6} 5 d^{2} P_{1 / 2}$ & $4 f^{4}\left({ }_{3}^{3} F\right) 5 p^{5}{ }^{4} D_{1 / 2}$ & 99613 & 1003.88 & $9.090 \mathrm{E}-05$ & $2.750 \mathrm{E}-05$ & $9.102 \mathrm{E}+04$ & 0.448 \\
\hline $4 f^{2}\left({ }_{1}^{3} F\right) 5 p^{6} 5 d^{2} P_{1 / 2}$ & $4 f^{4}\left({ }_{4}^{3} F\right) 5 p^{5}{ }^{4} D_{1 / 2}$ & 103718 & 964.15 & $2.294 \mathrm{E}-06$ & $7.228 \mathrm{E}-07$ & $2.593 \mathrm{E}+03$ & 0.625 \\
\hline $4 f^{2}\left({ }_{1}^{3} F\right) 5 p^{6} 5 d^{2} P_{1 / 2}$ & $4 f^{4}\left({ }_{3}^{1} D\right) 5 p^{5}{ }^{2} P_{1 / 2}$ & 105216 & 950.42 & $5.732 \mathrm{E}-04$ & $1.832 \mathrm{E}-04$ & $6.764 \mathrm{E}+05$ & 0.229 \\
\hline $4 f^{2}\left({ }_{1}^{3} F\right) 5 p^{6} 5 d^{2} P_{1 / 2}$ & $4 f^{2}\left({ }_{1}^{1} D\right) 5 p^{6} 6 p^{2} P_{1 / 2}$ & 108119 & 924.90 & $1.258 \mathrm{E}-02$ & $4.132 \mathrm{E}-03$ & $1.611 \mathrm{E}+07$ & 0.069 \\
\hline $4 f^{2}\left({ }_{1}^{3} F\right) 5 p^{6} 5 d^{2} P_{1 / 2}$ & $4 f^{4}\left({ }_{1}^{5} D\right) 5 p^{5}{ }^{6} D_{1 / 2}$ & 109048 & 917.03 & $6.412 \mathrm{E}-04$ & $2.124 \mathrm{E}-04$ & $8.424 \mathrm{E}+05$ & 0.310 \\
\hline $4 f^{2}\left({ }_{1}^{3} F\right) 5 p^{6} 5 d^{2} P_{1 / 2}$ & $4 f^{2}\left({ }_{1}^{3} P\right) 5 p^{6} 6 p^{4} D_{1 / 2}$ & 109666 & 911.85 & $4.762 \mathrm{E}-06$ & $1.586 \mathrm{E}-06$ & $6.363 \mathrm{E}+03$ & 0.182 \\
\hline $4 f^{2}\left({ }_{1}^{3} F\right) 5 p^{6} 5 d^{2} P_{1 / 2}$ & $4 f^{2}\left({ }_{1}^{3} P\right) 5 p^{6} 6 p^{2} S_{1 / 2}$ & 110081 & 908.41 & $5.629 \mathrm{E}-05$ & $1.882 \mathrm{E}-05$ & $7.608 \mathrm{E}+04$ & 0.611 \\
\hline $4 f^{2}\left({ }_{1}^{3} F\right) 5 p^{6} 5 d^{2} P_{1 / 2}$ & $4 f^{4}\left({ }_{1}^{5} D\right) 5 p^{5} \quad{ }^{6} D_{1 / 2}$ & 110468 & 905.24 & $1.101 \mathrm{E}-03$ & $3.695 \mathrm{E}-04$ & $1.503 \mathrm{E}+06$ & 0.170 \\
\hline $4 f^{2}\left({ }_{1}^{3} F\right) 5 p^{6} 5 d^{2} P_{1 / 2}$ & $4 f^{4}\left({ }_{1}^{3} D\right) 5 p^{5}{ }^{4} D_{1 / 2}$ & 112421 & 889.51 & $1.612 \mathrm{E}-03$ & $5.508 \mathrm{E}-04$ & $2.321 \mathrm{E}+06$ & 0.089 \\
\hline $4 f^{2}\left({ }_{1}^{3} F\right) 5 p^{6} 5 d^{2} P_{1 / 2}$ & $4 f^{4}\left({ }_{0}^{5} S\right) 5 p^{5}{ }^{4} P_{1 / 2}$ & 113266 & 882.87 & $1.875 \mathrm{E}-04$ & $6.451 \mathrm{E}-05$ & $2.760 \mathrm{E}+05$ & 0.153 \\
\hline $4 f^{2}\left({ }_{1}^{3} F\right) 5 p^{6} 5 d^{2} P_{1 / 2}$ & $4 f^{2}\left({ }_{1}^{3} P\right) 5 p^{6} 6 p{ }^{4} P_{1 / 2}$ & 115617 & 864.92 & $3.828 \mathrm{E}-03$ & $1.344 \mathrm{E}-03$ & $5.994 \mathrm{E}+06$ & 0.192 \\
\hline $4 f^{2}\left({ }_{1}^{3} F\right) 5 p^{6} 5 d^{2} P_{1 / 2}$ & $4 f^{4}\left({ }_{2}^{3} F\right) 5 p^{5}{ }^{4} D_{1 / 2}$ & 116976 & 854.87 & $6.502 \mathrm{E}-05$ & $2.310 \mathrm{E}-05$ & $1.054 \mathrm{E}+05$ & 0.454 \\
\hline $4 f^{2}\left({ }_{1}^{3} F\right) 5 p^{6} 5 d^{2} P_{1 / 2}$ & $4 f^{2}\left({ }_{1}^{3} P\right) 5 p^{6} 6 p^{2} P_{1 / 2}$ & 118179 & 846.17 & $3.156 \mathrm{E}-03$ & $1.133 \mathrm{E}-03$ & $5.277 \mathrm{E}+06$ & 0.251 \\
\hline $4 f^{2}\left({ }_{1}^{3} F\right) 5 p^{6} 5 d^{2} P_{1 / 2}$ & $4 f^{4}\left({ }_{2}^{3} P\right) 5 p^{5}{ }^{4} P_{1 / 2}$ & 119038 & 840.07 & $5.618 \mathrm{E}-03$ & $2.031 \mathrm{E}-03$ & $9.600 \mathrm{E}+06$ & 0.113 \\
\hline
\end{tabular}

Note - Table 14 is published in its entirety in the machine-readable format. Part of the values are shown here for guidance regarding its form and content. 